\title{
1 Organization of cortico-hippocampal networks in the human brain
}

3 Short Title: Organization of cortico-hippocampal networks

5 Alexander J. Barnett*1, Walter Reilly ${ }^{1}$, Halle R. Dimsdale-Zucker ${ }^{2}$, Eda Mizrak ${ }^{1,3}$, Zachariah Reagh ${ }^{1,4,5}$, Charan

6 Ranganath ${ }^{1}$

8 1. University of California, Davis, Center for Neuroscience, 1544 Newton Ct, Davis, CA 95618, United States

9 2. Columbia University, Department of Psychology, 312 Schermerhorn Hall 1190 Amsterdam Avenue New York, $10 \quad$ NY 10027

11 3. University of Zurich, Department of Psychology, Binzmühlestrasse 14, 8050 Zürich, Switzerland

12 4. University of California, Davis, Department of Neurology, 3160 Folsom Blvd., Suite 2100, Sacramento, CA

1395816

14 5. Washington University in St. Louis, Department of Psychological and Brain Sciences, 1 Brookings Dr.

15 St. Louis, MO 63130

16

17 *Corresponding Author

18 Alexander J. Barnett

191544 Newton Ct, Davis, CA 95618, United States

20 Center for Neuroscience, Room 209

21 Email: ajbarnett@ucdavis.edu

23 Alexander J. Barnett: https://orcid.org/0000-0001-5891-7880

24 Walter Reilly: https://orcid.org/0000-0003-2303-3094

25 Halle Dimsdale-Zucker: https://orcid.org/0000-0002-8611-111X

26 Eda Mizrak: https://orcid.org/0000-0002-1765-7047

27 Zachariah Reagh: https://orcid.org/0000-0002-4763-4268

28 Charan Ranganath: https://orcid.org/0000-0001-5835-6091

30 Author Contributions

31 AJB, WR, HDZ, and CR designed research; AJB, HDZ, EM, and ZR performed research; EM contributed

32 new reagents or analytic tools; AJB and WR analyzed data. AJB and CR wrote the paper 


\section{Abstract}

34 Episodic memory depends on interactions between the hippocampus and the interconnected

35 regions comprising default mode network (DMN). Here, using data-driven analyses of resting-

36 state fMRI data, we identified the networks that interact with the hippocampus - the DMN and a

37 "Medial Temporal Network" (MTN) that included regions in the medial temporal lobe and

38 retrosplenial cortex. We observed that the MTN plays a critical role in connecting the visual

39 network to the DMN and hippocampus. The DMN could be further divided into three

40 subnetworks: a "Posterior-Medial" Subnetwork comprised of posterior cingulate, and lateral

41 parietal cortices, an "Anterior-Temporal" Subnetwork comprised of regions in the temporopolar,

42 and dorsomedial prefrontal cortex, and a "Medial-Prefrontal" Subnetwork comprised of regions

43 primarily in the medial prefrontal cortex. These networks vary in their functional connectivity

44 along the hippocampal long-axis and represent different kinds of information during memory-

45 guided decision-making. Finally, a Neurosynth meta-analysis of fMRI studies suggests new

46 hypotheses regarding the functions of the MTN and DMN subnetworks, providing a framework

47 to guide future research on the neural architecture of episodic memory. 
48 Episodic memory allows us to relive past events that happened at a particular place and time (1).

49 Most research investigating the neurobiology of episodic memory retrieval has focused on the

50 hippocampus and medial temporal lobe (MTL) cortex (perirhinal (PRC), parahippocampal

51 (PHC), and entorhinal cortex) highlighting the interactions among these regions (2-4). It is

52 generally assumed, however, that the hippocampus supports memory by integrating information

53 represented across distributed areas in the neocortex (5). Consistent with this idea, fMRI studies

54 of memory retrieval have shown activity and connectivity in a cortico-hippocampal network

55 composed of medial parietal, lateral parietal, lateral temporal, and medial prefrontal neocortical

56 regions along with the MTL (6-10), and lesion studies have shown that damage within this

57 network can cause amnesia (11).

58 This distributed set of cortical regions that are recruited during episodic retrieval overlaps

59 extensively with regions that comprise the default-mode network (DMN). The DMN is a large-

60 scale network that is typically identified in studies that use intrinsic functional connectivity

61 analysis of functional magnetic resonance imaging (fMRI) data (12), and some studies suggest

62 that the DMN can be differentiated into different subnetworks (13-15). One approach to partition

63 the DMN has been to view DMN subnetworks as an extension of the connectivity differences

64 within the MTL (16-18). Guided by neuroanatomical studies of MTL connectivity in rodents and

65 nonhuman primates (19-22), studies of intrinsic functional connectivity in fMRI data (23-26)

66 have differentiated between cortical regions that preferentially affiliate with the PRC and anterior

67 hippocampus and cortical regions that preferentially affiliate with the PHC and posterior

68 hippocampus $(27,28)$. For example, Libby et al. (24) demonstrated that the PHC has relatively

69 higher functional connectivity with a network of Posterior Medial (PM) cortical regions,

70 including the posterior cingulate and retrosplenial cortex and posterior hippocampus, whereas the 
71 PRC has relatively higher connectivity with a network of Anterior Temporal (AT) cortical

72 regions, including the orbitofrontal and temporopolar cortex and anterior hippocampus. Drawing

73 on these findings, Ranganath and Ritchey (29) reviewed evidence converging on the idea that

74 PM and AT networks support different aspects of memory-guided behavior. This "PM/AT

75 framework" has provided a valuable framework for interpreting memory phenomena in imaging

$76(9,30-32)$, stimulation $(33,34)$, and disease $(35-37)$. However, recent work has come to

77 question the homogeneity of the PM network (38), with several studies highlighting a

78 dissociation between PHC, retrosplenial and precuneal cortex from posterior cingulate cortex

79 (PCC) $(39,40)$ under conditions that seem to tax perceptual relative to abstract event processing,

80 or scene relative to face processing.

81 A second approach to segmenting the DMN has used data-driven methods and these

82 studies have also found partially conflicting network delineations $(15,41,42)$. These studies

83 have generally partitioned the DMN into three subnetworks: an MTL subnetwork, a midline

84 subnetwork of posterior cingulate and medial prefrontal cortex, and a third network composed of

85 dorsal medial prefrontal, lateral temporal, and ventrolateral prefrontal cortex, but have focussed

86 largely on cortical regions. Critically, it is unclear how these data-driven, cortical networks

87 pertain to memory functioning and previous frameworks that have strongly characterized

88 hippocampal connections.

89 Here, we seek to unify these two approaches. The MTL-centric approach has rarely

90 considered the interplay of regions outside of the MTL, and a recent review has suggested that

91 considerable heterogeneity may exist within the previously described PM network (38),

92 prompting a need for re-examination of these network properties. Conversely, the data-driven

93 approach has rarely described the hippocampal interactions with these networks, focusing mainly 
94 on cortico-cortical interactions limiting the applicability to understanding episodic memory. The

95 goal of this paper was to comprehensively characterize cortico-hippocampal networks that

96 contribute to episodic memory. Using a whole-brain, data-driven approach to examine functional

97 connectivity in resting-state fMRI, we sought to 1 . identify and partition the DMN into

98 subnetworks and examine whether these subnetworks converge with the PM/AT framework, 2.

99 examine the connectivity of the hippocampus to the identified networks, and 3. determine

100 whether these cortico-hippocampal networks play different roles in episodic memory.

101

102 Results

103 Data-Driven Partition of Neocortical Networks

104 Our first objective was to use a data-driven approach to identify large-scale resting-state

105 networks. Using 25 minutes of resting-state fMRI data acquired from 40 participants, we

106 partitioned the brain into canonical resting networks using state-of-the-art techniques (43). We

107 extracted the average confound-corrected timeseries from each region in a recently published

108 atlas of the human neocortex (44) and computed Fisher z-transformed Pearson's correlations

109 between the timeseries of each cortical region and every other cortical region. We then created a

110 group-averaged functional connectivity matrix to be used for community detection. The Louvain

111 community detection algorithm (45) was run for 1000 iterations, tuning the resolution (how large

112 or small communities might be) across a range of resolution parameters to identify a partition

113 solution that showed both high network modularity (i.e. higher within community connectivity

114 than would be expected by chance) and high network grouping consistency (quantified using the

115 z-Rand index $(43,46))$. The solution was also subject to a qualitative criterion that the partition

116 should separate the primary sensory networks (43). The resolution parameter of gamma $=2.005$ 
117 satisfied our criteria and produced a network partition (Figure 1) that corresponds closely with

118 previously reported partitions in other recent studies $(43,47,48)$. This partition identified the

119 DMN, but, interestingly, some areas (such as the PHC, retrosplenial cortex, and PRC) that are

120 often identified as part of the DMN were grouped within a separate network that included MTL

121 and parietal regions. This set of regions overlaps with what has been previously described as the

122 MTL network (13), or the contextual association network (49), and coactivates particularly

123 during recall of places $(50,51)$. Here we will describe this network as the medial temporal

124 network (MTN).
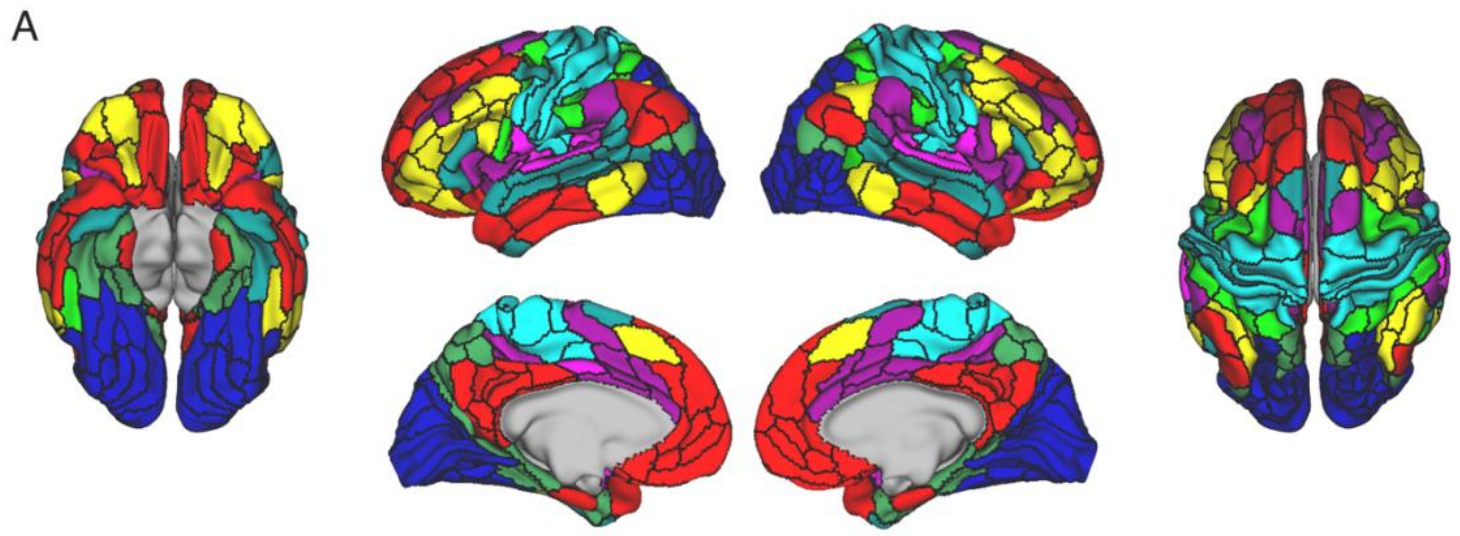

B
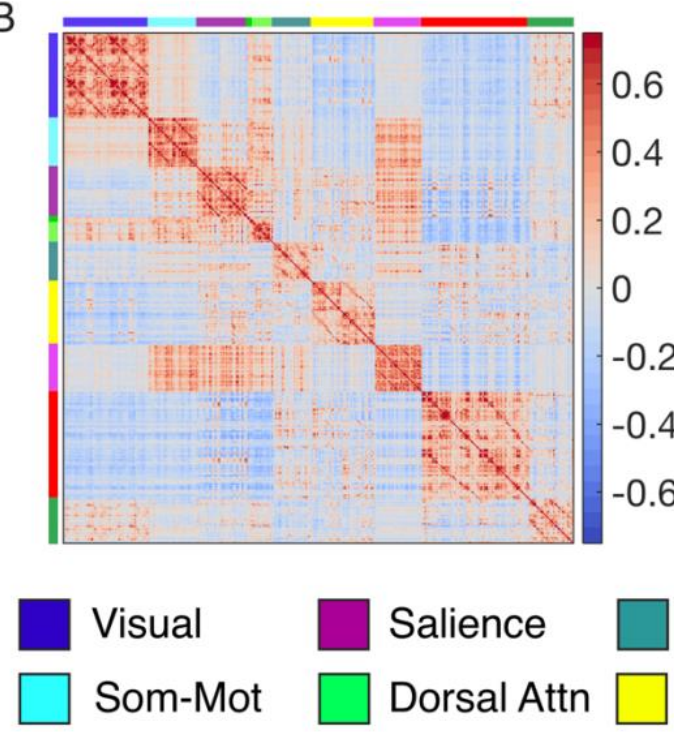

C

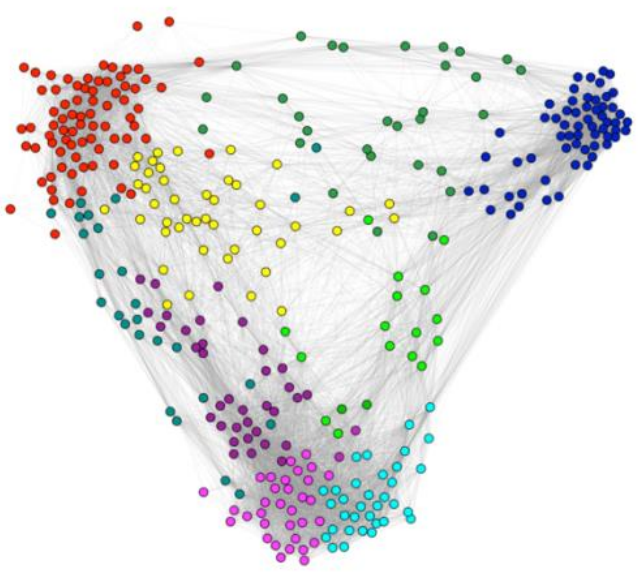

Language Front Par

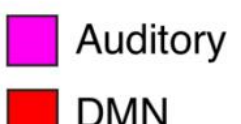


127 Figure 1. Louvain community detection identifies large-scale resting-state networks including

128 the default-mode and medial temporal networks that are thought to interface with the

129 hippocampus. A. Inflated cortical surface, colored according to community membership. B.

130 Connectivity matrix reordered by community to demonstrate the community structure of the

131 group-averaged brain. Colors along the axis demonstrate which rows/columns belong to a given

132 community. Color bar represents Fisher Z-transformed correlation values. C. Force-directed

133 graph of the group-averaged networks color coded by community membership of the selected

134 partition created using the ForceAtlas2 algorithm (52). Attn, attention; DMN, Default mode

135 network; Front Par, Frontoparietal; MTN, Medial temporal network; Som-Mot, Somatomotor.

137 We next interrogated the functional connectivity between the networks defined above and the

138 hippocampus. We segmented the hippocampus in each participant using FreeSurfer v6.0

139 (http://surfer.nmr.mgh.harvard.edu/, (53)), and then manually segmented it further into anterior

140 and posterior regions, as these subdivisions of the hippocampus are known to have somewhat

141 different functional properties $(16,20)$. The posterior hippocampus was defined as all the

142 hippocampus posterior to the last slice of the uncal notch (16). We calculated the connectivity of

143 the hippocampus to every cortical region in the atlas and averaged together functional

144 connectivity weights of cortical regions within the same network based on network affiliations.

145 This was done for each participant. One-sample t-tests revealed that two networks were

146 functionally connected to every hippocampal ROI in our sample - the DMN and the MTN

147 (DMN range: $t(38)=10.7-12.9$, all $P<.001$; MTN range: $t(38)=4.2-9.3$, all $P<.01)$. The

148 language network and somatomotor network both showed mixed connectivity with the

149 hippocampus, showing significant anterior, but not posterior hippocampal connectivity (see 
150 Supplemental Table 1). The robust connectivity of the MTN and DMN to the hippocampus

151 corroborates previous investigations of hippocampal connectivity (54).

153 Network analysis support an interfacing role of the MTN

154 A recent review discussing the heterogeneity within the PM network suggested that

155 parahippocampal, and lateral parietal cortex interface with lower level sensory cortex in service

156 of higher-order feature encoding prior to binding in the hippocampus $(29,38)$. They also note

157 that these regions also interface with other DMN regions such as the $\mathrm{MPFC}$ and PCC (regions

158 found in the DMN, here) that track long-timescale event structure and provide conceptual

159 knowledge pertaining to event schemas $(38,55)$. Thus, the MTN may serve as a bridge between

160 external, low level perception in the visual network and the hippocampus and DMN. By plotting

161 the structure of the network interactions in Figure 2A (left) we do indeed see that the MTN

162 shows functional connectivity with both the DMN, hippocampus, and the visual network.

163 Treating the brain as an abstract graph (56), we sought to test the hypothesis that the MTN

164 mediates network communication between the visual network and the DMN and hippocampus.

165 To do so, we used graph theory to calculate the shortest path length between the visual network

166 and the DMN and hippocampus. Path length refers to the shortest number of links that needs to

167 be traversed to link one node in a network to a target node. If two regions are directly connected

168 by a strong link, they will have a short path length. If two regions are not directly connected by a

169 positive link but can be connected by multiple intermediate nodes that are positively connected,

170 then they would have a longer path length. To examine the influence of the MTN on

171 internetwork communication between the DMN and visual network, we compared the path

172 length between the visual network and DMN when the MTN was removed relative to the path 
173 length when we removed all other networks. If the MTN mediates the connectivity between the

174 visual network and DMN and hippocampus, then removal of the MTN should disproportionately

175 increase the average path length between visual network and DMN over and above any path

176 length increases caused by removal of all other networks. This was done at the individual-subject

177 level to determine the significance of the effect and is similar to methods used by (57).

178 We found that across subjects, removal of the MTN led to a disproportionately large

179 increase in DMN-visual network path length compared to removal of any other network (all

$180 t(39)>6.7, \mathrm{p}<.001$; Figure 2B), indicating that the MTN plays a critical role in cross-network

181 communication between the visual network and DMN. Importantly, this effect cannot be

182 explained simply by the number of nodes removed from the whole network, as the MTN has

183 fewer nodes (30 nodes) than the somatomotor (34 nodes), salience (35 nodes), frontoparietal (44

184 nodes) and auditory (33 nodes) networks. To further confirm this, we randomly removed 30

185 nodes from each subject's network (excluding DMN, and visual nodes) and recalculated the path

186 length values. This was performed 1000 times and we compared the DMN-visual network path

187 length of the true removal of the MTN nodes compared to the distribution of DMN-visual

188 network path length following random node removals. We observed that the MTN removal still

189 resulted in greater average path length between the DMN and visual network relative to removal

190 of random nodes, $t(39)=9.4, p<.001$. Another possible explanation is that due to the high level

191 of connectivity between the DMN and MTN, removal of the MTN will non-specifically lead to a

192 disproportionate increase in path length of the DMN to all other networks. However, the path

193 length effects caused by removal of the MTN were specific to the DMN - visual network path

194 lengths (S1 Figure). 


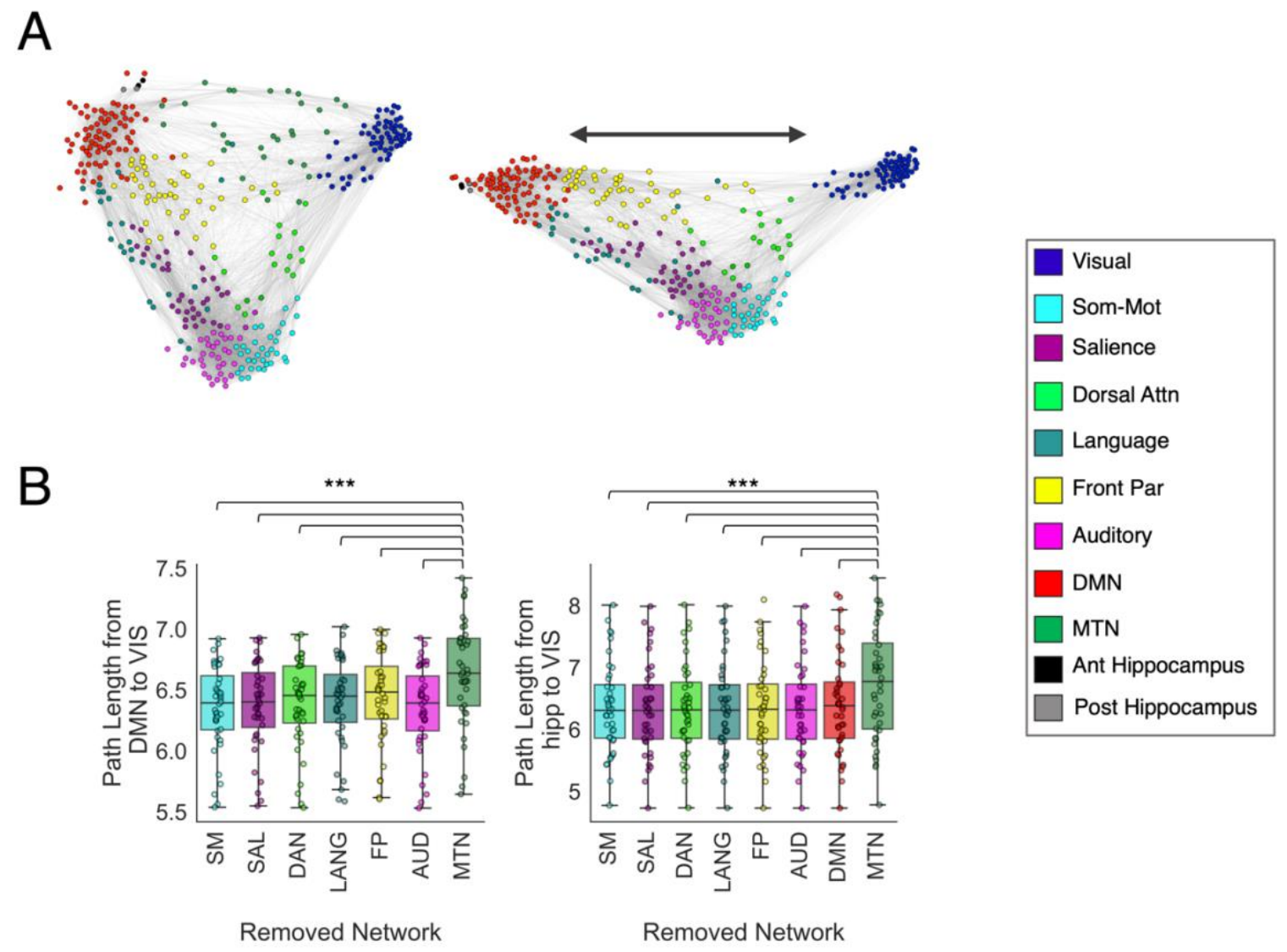

196 Figure 2. Removal of the MTN disproportionately decreases the network connectivity of the

197 DMN and visual network. (A) Force-directed graph of the group-averaged networks color coded

198 by community membership of the selected partition created using the ForceAtlas 2 algorithm (52)

199 with the MTN present (left) and with the MTN excluded (right). (B) Boxplots displaying the

200 path length between the DMN and visual network or the path length between the hippocampi and

201 visual network following removal of each network. $* * * \mathrm{p}<.001$

As mentioned above, it is well-known that MTL regions such as the perirhinal and

204 parahippocampal cortex provide the hippocampus with higher-order representations of objects

205 and scenes $(4,58)$. Therefore, we repeated the previous analysis, this time examining the effects

206 of MTN removal on the pathlength between the hippocampus and visual network. We observed 
207 that removal of the MTN lead to greater path length between the hippocampus and visual

208 network compared to removal of any other networks (all $t(39)>6, \mathrm{p}<.001$; Figure 2B). Again,

209 MTN removal led to disproportionately higher path length than removal of random nodes $(t(39)$

$210=3.15, p=.003$ ). This effect was specifically between the hippocampus and visual network path

211 length, rather than leading to disproportionately higher path length between the hippocampus and

212 any other network, S2 Figure.

\section{Partitioning the DMN}

215 We next sought to identify whether the DMN could be broken up into subnetworks using similar

216 methods that we used in our whole-brain network partition. We selected the DMN regions

217 identified in our whole-brain partition and performed Louvain community detection on the

218 functional connections between the regions in the network. This was done for 1000 iterations

219 across a range of resolution parameters, and we again selected the partition yielding the highest

220 modularity and stability. The solution with the highest modularity-weighted stability produced a

221 partition of the DMN with three communities which we refer to as the PM subnetwork, the AT

222 subnetwork and the medial prefrontal (MP) subnetwork. The PM subnetwork and AT

223 subnetwork were named as such based on their resemblance to previous spatial maps of these

224 networks (24). The PM subnetwork included posterior cingulate cortex, posterior angular gyrus,

225 and dorsal prefrontal cortex. The AT subnetwork included temporopolar cortex, lateral

226 orbitofrontal cortex, temporoparietal junction and dorsomedial prefrontal cortex. The MP

227 subnetwork included medial prefrontal regions and the entorhinal cortex (Figure 3). 


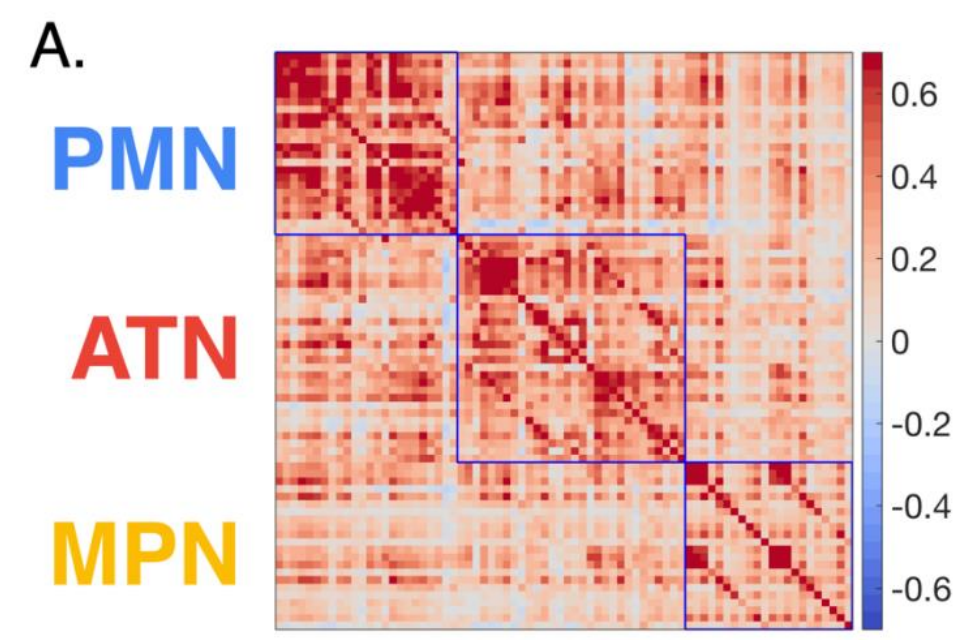

B.
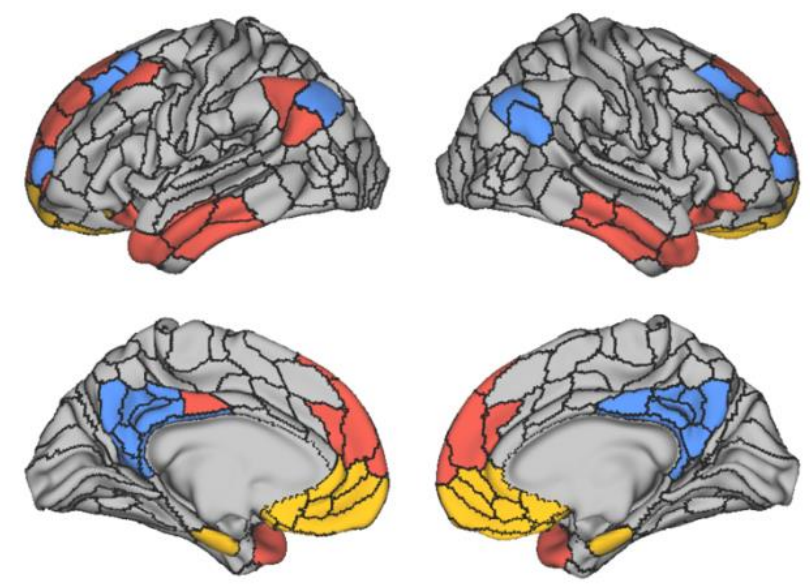

231 Figure 3. The default-mode network can be partitioned into three interconnect subnetworks. A.

232 Functional connectivity matrix organized by subnetwork. Color bar represents Fisher Z-

233 transformed correlation values. B. Inflated cortical surface, colored according to community

234 membership of subnetworks. AT, anterior temporal; MP, medial prefrontal; PM, posterior

235 medial.

237 To visualize the topology of connections between these DMN sub-networks and the other large-

238 scale networks defined earlier, we constructed a force-directed graph in abstract graph space 
239 (Figure 4). The ForceAtlas2 algorithm was used to construct the layout by modeling the graph as

240 a physical system in which nodes repel each other and the functional connectivity between the

241 nodes act as springs (52). Regions within the PM subnetwork tightly clustered and several nodes

242 showed prominent cross-network connectivity with the MTN and frontoparietal network. The

243 AT subnetwork showed cross-network interactions with the language and frontoparietal network

244 and the MP subnetwork was less clustered with fewer out of DMN connections. 


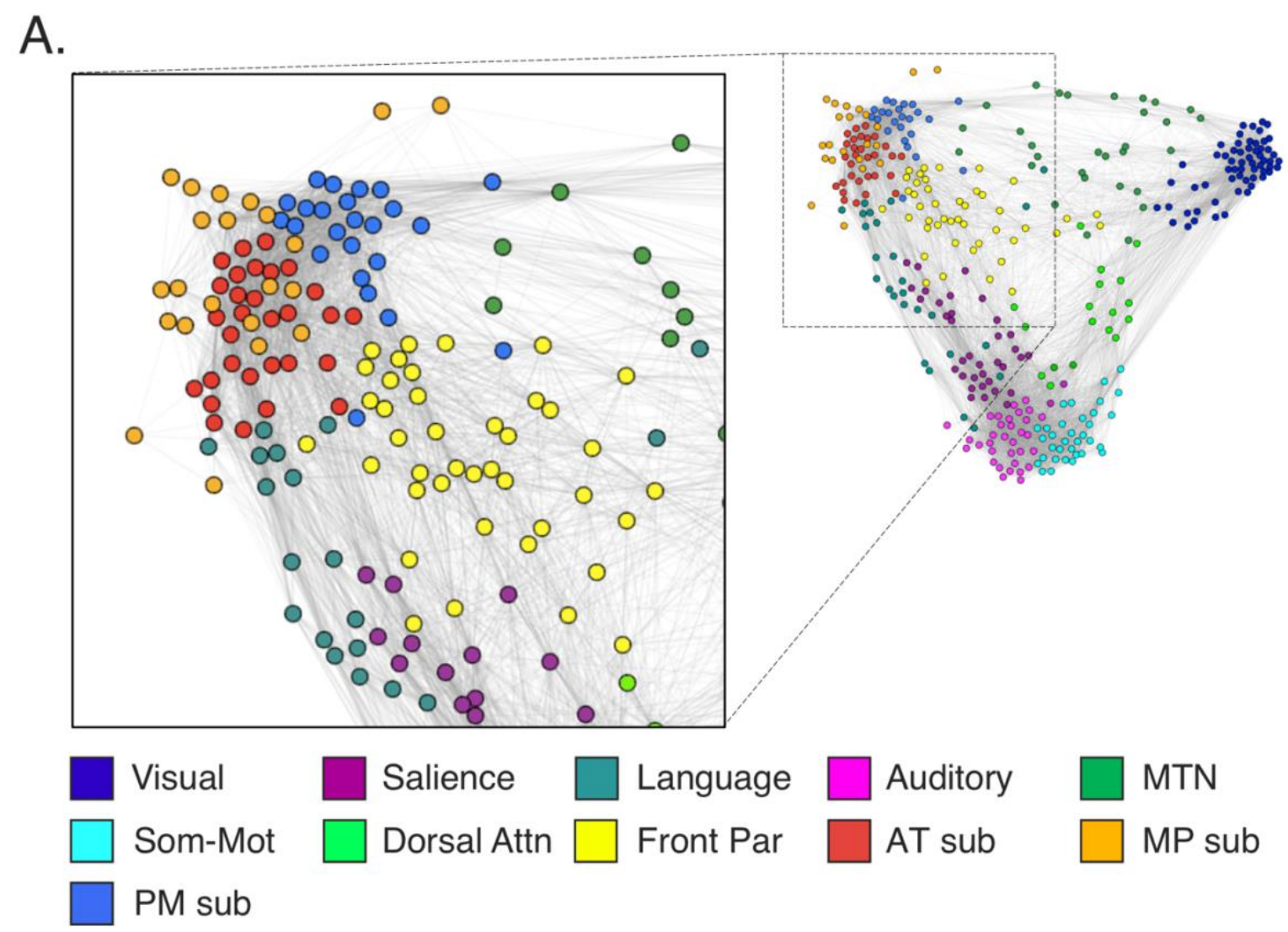

B.

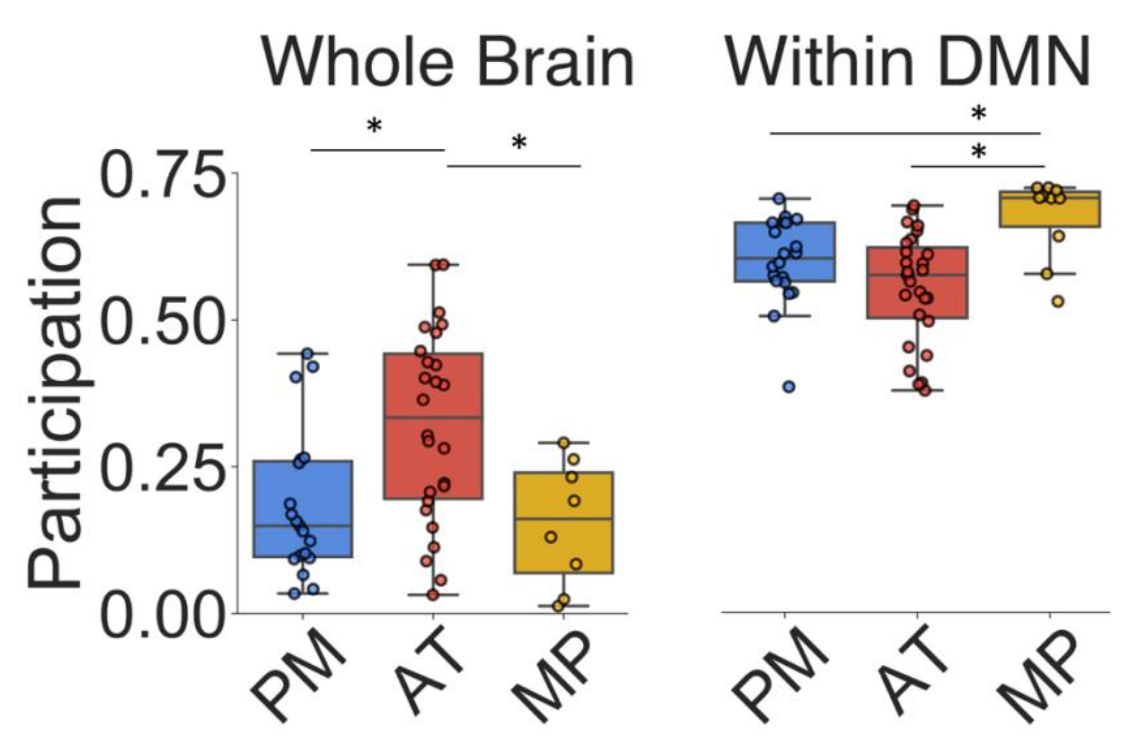

247 Figure 4. The subnetworks of the DMN interact with language, frontoparietal and medial

248 temporal networks. A. Force-directed graph of the group-averaged networks with the DMN

249 relabelled according to subnetwork membership. B. Boxplots showing participation coefficient 
250 for DMN nodes to the rest of the brain (Whole Brain) and participation coefficient for DMN

251 nodes to subnetworks of the DMN (Within DMN). AT, anterior temporal; Attn, attention; Front

252 Par, Frontoparietal; MP, medial prefrontal; MTN, medial temporal network; PM, posterior

253 medial; Som-Mot, Somatomotor. *indicates significant difference at $\mathrm{p}<.05$.

255 To quantify the diversity of connections in the DMN subnetworks to the rest of the brain, we 256 calculated participation coefficients - a graph theory metric that describes the extent to which a

257 node's functional connections are spread across different communities. Nodes with a high

258 participation coefficient are thought to be critical for communication between communities, and

259 disruption of such nodes can lead to altered network function and cognitive impairment (59-62).

260 Of the subnetworks, the AT subnetwork had the greatest diversity of connections to nodes

261 outside of the DMN (AT > PM: $t(43)=3, P=.004$; AT > MP: $t(32)=2.7, P=.01)$. As shown in

262 Figure 4B, the PM subnetwork had several nodes with relatively high participation. These nodes

263 connect to MTN, and the frontoparietal network, but the majority of the PM subnetwork showed

264 less out of network connectivity. The MP subnetwork, on the other hand, had relatively low

265 participation outside of the DMN.

We next addressed the extent to which nodes in the DMN showed diverse connectivity

267 with nodes in the other DMN subnetworks. Interestingly, regions in the MP subnetwork

268 exhibited the highest participation coefficients across the DMN subnetworks (MP > PM: $t(28)=$

$2692.7, P=.01 ;$ MP $>$ AT: $t(35)=3.7, P<.0007)$. These findings substantiate what can be seen in

270 Figure 4A - that nodes in the MP subnetwork are positioned to integrate information across

271 DMN subnetworks, whereas nodes in the AT subnetwork and PM subnetwork may interface

272 with networks outside of the DMN. 
274 Cortico-hippocampal network connectivity

275 Having identified cortical networks and subnetworks that connect to the hippocampus (cortico-

276 hippocampal networks), we then examined whether these networks showed a preference in

277 connectivity along the hippocampal long-axis. Previous research has shown differential

278 functional connectivity along the long-axis axis of the hippocampus $(24,28,63$, 64), with the

279 anterior hippocampus demonstrating greater connectivity to orbitofrontal cortex, and temporal

280 pole and the posterior hippocampus demonstrating greater connectivity to retrosplenial cortex

281 and precuneus. We therefore hypothesized that the anterior hippocampus should have

282 preferential connectivity to the AT subnetwork, whereas the posterior hippocampus would have

283 preferred connectivity with the PM subnetwork. These predictions were partially confirmed by

284 our findings. The anterior hippocampus showed stronger connectivity to the AT and MP

285 subnetworks $(t(37)=8.2, P<.00001 ; t(37)=6.5, P<.00001)$ and the posterior hippocampus

286 showed stronger connectivity to the MTN than the anterior hippocampus $(t(37)=2.6, P=.01)$,

287 but there was no significant difference in connectivity between the hippocampal regions and PM

288 subnetwork $(t(37)=.41, P=.68)($ Figure $5 \mathrm{~A})$. To determine whether these differences were

289 evenly spread across the networks or driven by particular regions within the networks, we

290 contrasted anterior and posterior hippocampal connectivity at a region-to-region level. As

291 expected from the network-level analysis, we observed significantly greater anterior

292 hippocampal connectivity to medial prefrontal, anterior temporal, and also anterior medial

293 temporal cortex, whereas the posterior hippocampus had significantly greater connectivity to the

294 parietal occipital sulcus, precuneus, and dorsal posterior cingulate cortex. Thus, the anterior-

295 posterior differences are fairly consistent in the AT and MP subnetworks, but within the MTN 
296 the overall anterior-posterior differences are driven by relatively higher posterior hippocampal

297 connectivity in the medial parietal cortex (Figure 5B).

298 Based on neuropsychological evidence demonstrating distinct functional roles of the left

299 and right hippocampus (65-67), we next investigated whether the left and right hippocampus

300 differed in their connectivity to the cortical networks. We observed significant hemispheric

301 laterality effects with the right hippocampus having greater connectivity to the MTN than the left

$302(t(37)=3.8, P=.0001)$ and the left hippocampus having greater connectivity to AT subnetwork

$303(t(37)=2.6, P=.01)$. There was no significant laterality effect in the PM $(t(37)=.29, P=.77)$

304 and MP subnetworks $(t(37)=.98, P=.33)$. This connectivity difference might help to explain

305 differentiated function of the left and right hippocampus. 


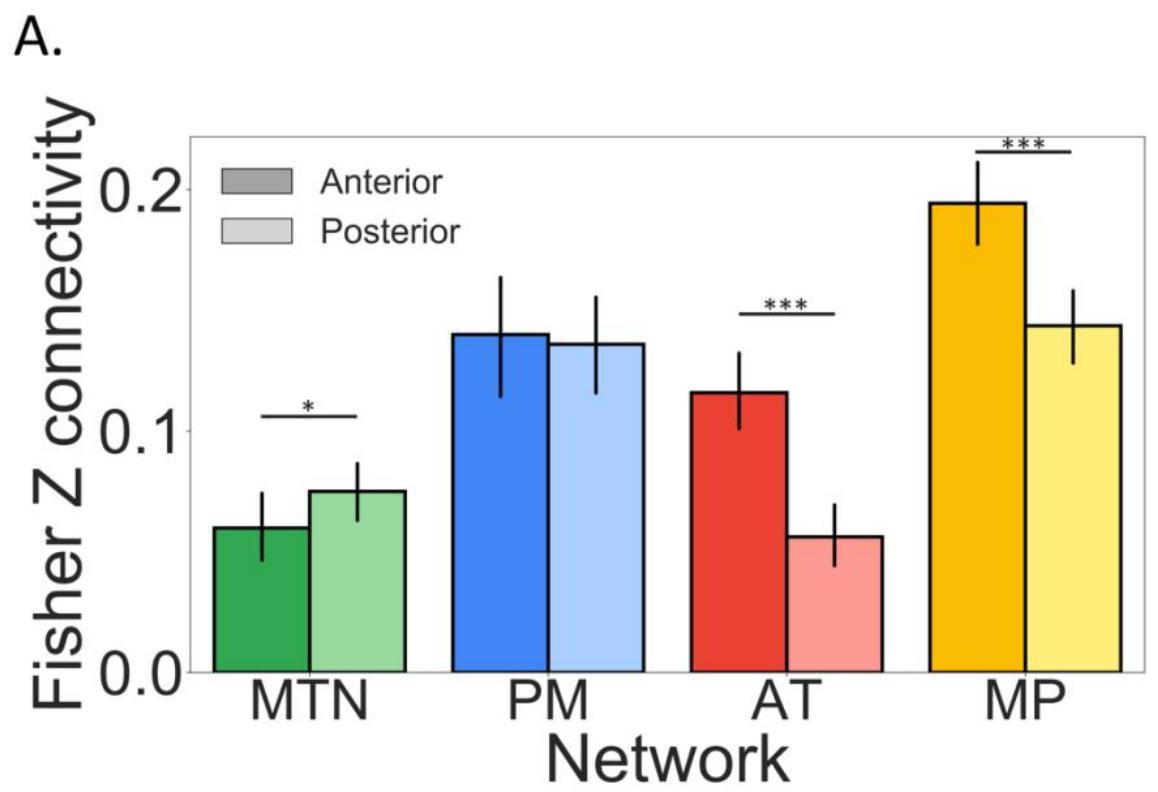

B.
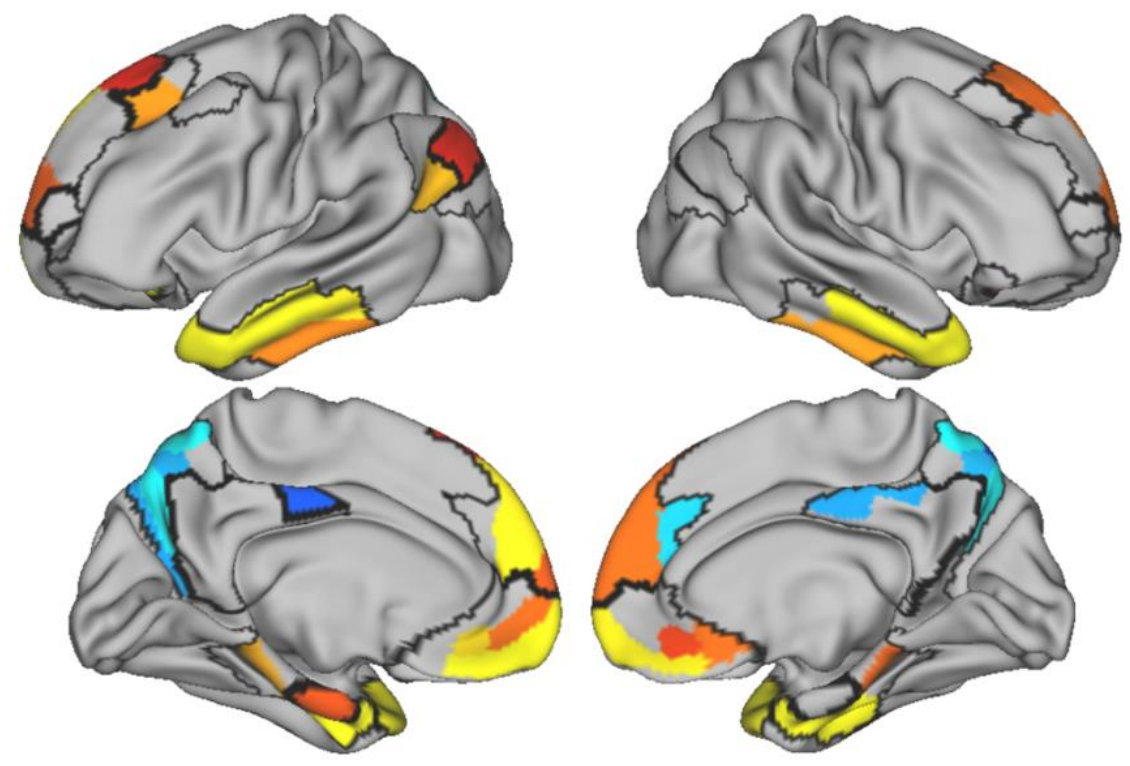

Post $\mathrm{HC}>$ Ant $\mathrm{HC}$

T-value
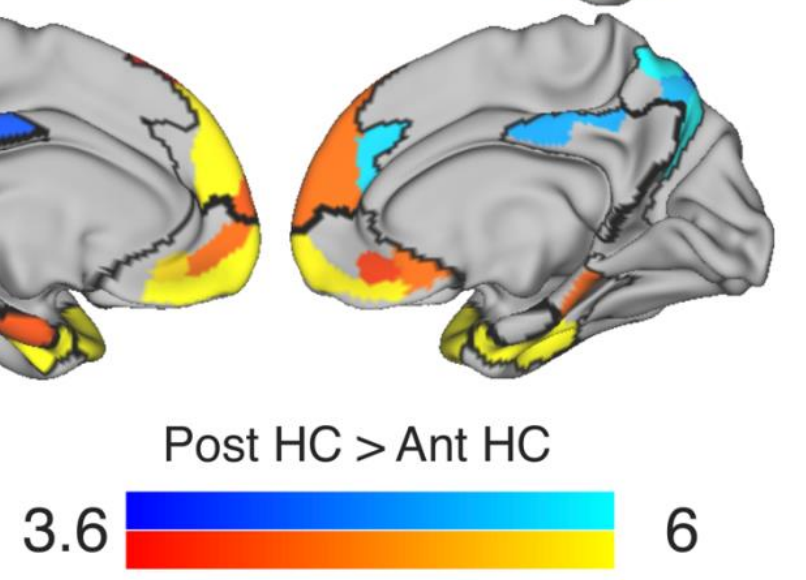

6

Ant $\mathrm{HC}>$ Post $\mathrm{HC}$

309 Figure 5. Regions within the MTN and DMN subnetworks exhibit differential functional

310 connectivity with the anterior and posterior hippocampus. A: Bar graphs of functional 
311 connectivity from each of the cortical networks to the anterior and posterior hippocampus. Error

312 bars show $95 \%$ confidence interval. B: T-contrast of anterior hippocampal connectivity versus

313 posterior hippocampal connectivity, with warm colors showing regions that had stronger anterior

314 hippocampal connectivity and cool colors showing regions that had stronger posterior

315 hippocampal connectivity. Cortico-hippocampal network boundaries are outlined on the surface

316 of the brain. These results indicate that the temporopolar and medial prefrontal cortex show

317 preferential connectivity with the anterior hippocampus, whereas posterior medial parietal cortex

318 shows preferential connectivity with the posterior hippocampus. Ant, anterior; AT, anterior

319 temporal; HC, hippocampus; MP, medial prefrontal; MTN, medial temporal network; PM,

320 posterior medial; Post, posterior. $* P<.05, * * * P<.001$.

322 Regions within the same community represent similar kinds of information during a memory

$323 \underline{\text { task }}$

324 We next sought to determine whether network membership was related to functional relevance

325 of these subnetworks using an independent dataset in which participants performed a memory

326 retrieval task (described in Mizrak et al. (68) and in Methods section) by examining the

327 representational profile similarity between regions within the same network or in different

328 networks $(17,69)$. Multivoxel pattern similarity was calculated between trials which created a

329 trial-by-trial representational similarity matrix for every ROI in our cortico-hippocampal

330 networks. Here, we assume that trial-by-trial fluctuations in pattern similarity for a given ROI

331 are driven by trial-by-trial fluctuations in features for which the ROI is sensitive. These

332 fluctuations across trials represent the ROI's representational profile. We sought to determine 
333 whether regions within the same network had greater similarity in their representational

334 profiles — and thus represent similar features — compared to regions outside their network.

335 Here, we calculated the similarity of representational profiles between ROIs by

336 correlating each ROI's representational similarity matrix to every other ROI. We then averaged

337 the representational profile similarities for pairs of ROIs within the same network to create a

338 mean within-network similarity value, for each subject (we removed the similarity of each ROI

339 with itself to avoid inflating the within network similarity). To create a mean between-network

340 similarity value, for each subject, we averaged together the representational profile similarity

341 values for pairings of ROIs that were in different networks. We contrasted the mean within-

342 network similarity with the mean between-network similarity using a repeated-measures

343 ANOVA and observed that regions within the same network had higher profile similarity

344 compared to regions in different networks, $t(21)=18.5, P<.001$ (Figure 6). This effect persists

345 even when accounting for the similarity in trial-by-trial mean BOLD activity $(t(21)=12.1, \mathrm{p}<$

$346.001)$, suggesting it is not simply a result of univariate activity similarity.
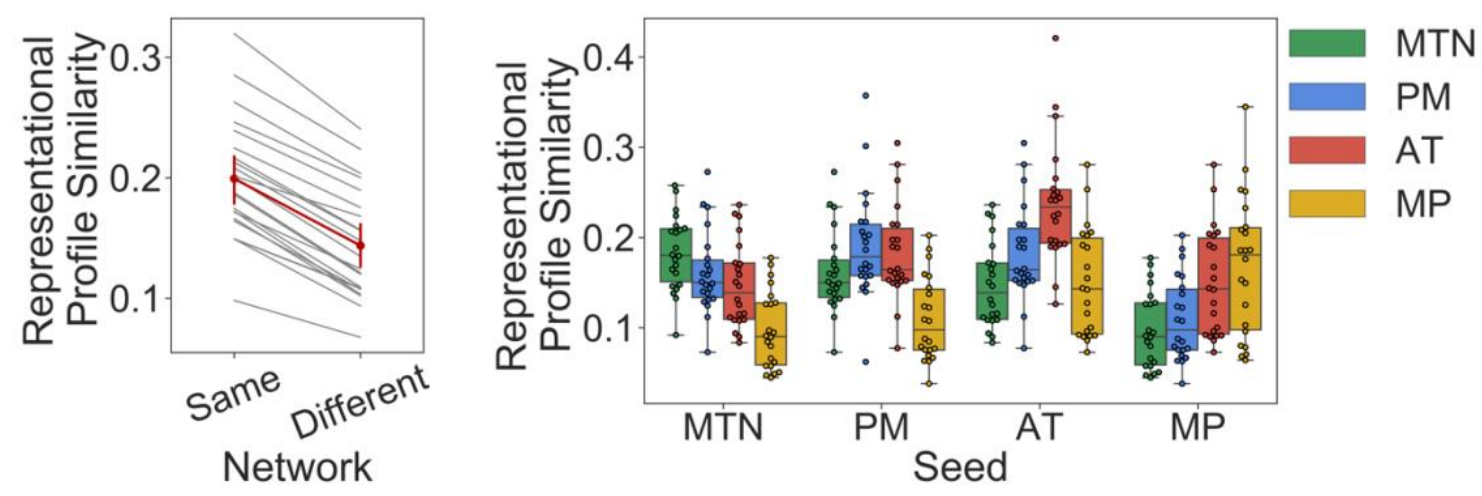

349 Figure 6. Regions show higher representational profile similarity with community members

350 compared to regions in other communities. Left: Individual subject representational profile 
351 similarity of regions within the same network or between network in grey lines with the group

352 average in red, with $95 \%$ confidence intervals represented by error bars. Right: Boxplot of

353 average region-to-region representational profile similarity between each cortical-hippocampal

354 network (labelled along the x-axis) to itself and every other cortico-hippocampal network (each

355 represented by a colored bar) across the sample. Individual participants represented as dots. AT,

356 anterior temporal; MP, medial prefrontal; MTN, medial temporal network; PM, posterior medial.

358 Another possible explanation of these findings is that regions in the same network tend to be

359 closer in spatial proximity and there is some spatial non-independence of adjacent ROIs of these

360 representational effects. To account for this spatial proximity, we created an ROI-by-ROI

361 distance matrix by calculating the Euclidean distance between the center of gravity of all pairs of

362 ROIs. We then regressed out the influence of spatial proximity from the representational profile

363 matrix using this distance matrix and repeated the analysis. After statistically removing the

364 influence of spatial proximity, we still observed that ROIs within the same network had greater

365 representational profile similarity compared to pairs of ROIs in different networks, $t(21)=9.3, p$

$366<.001$.

$367 \quad$ Neurosynth Decoding

368 Having found evidence that regions within the same cortico-hippocampal networks carry similar

369 information, we sought to identify the cognitive terms typically associated with activity in these

370 networks. To better understand the role these networks may play in cognition, we used

371 Neurosynth (70) to determine what cognitive terms are typically associated with activation in

372 these subnetworks. The meta-analytic activation map of every term in the Neurosynth database

373 was correlated with the binarized volumetric masks of each cortico-hippocampal network. By 
374 examining the top cognitive terms associated with each network we observed that the term

375 autobiographical was the sole common term in the top 20 across all networks. Activation in

376 MTN and PMN is disproportionately associated with studies involving episodic memory, but this

377 was not the case for the ATN and MPN in which the term episodic memory did not appear in the

378 top 20 search terms. The MTN also showed strong overlap with meta-analytic maps for

379 preference for navigation $(r=.15)$, scenes $(r=.12)$, and the PM subnetwork showed strong

380 overlap with self-referential $(r=.13)$, and theory of mind terms $(r=.08)$. The top terms

381 associated with AT subnetwork related to theory of mind $(r=.22)$, intentions $(r=.17)$, mental

382 states $(r=.22)$, and social cognition $(r=.19)$, while the top terms associated with MP

383 subnetwork were value $(r=.17)$, fear $(r=.16)$, emotion $(r=.16)$, and terms related to emotional

384 valence $(r=.14)$. Weightings for each network are available in Supplementary Table 2.
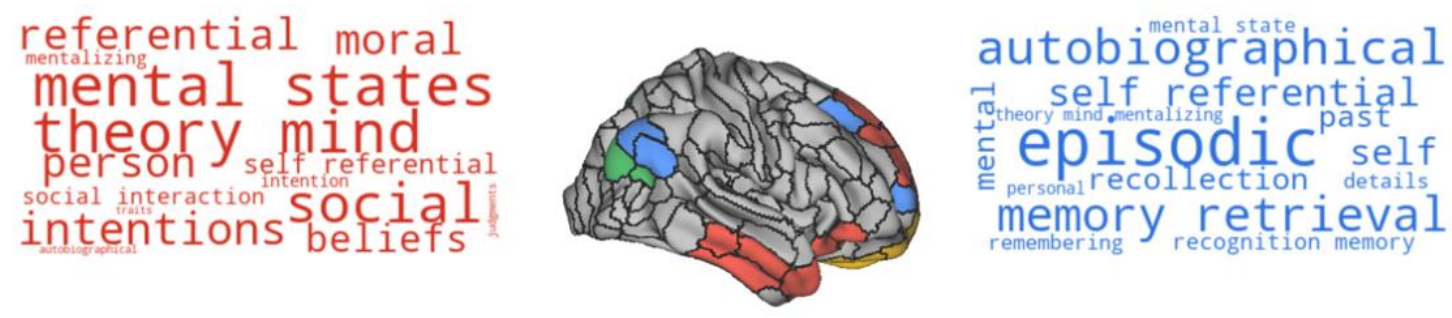

$\rightarrow$ self referential

Theory mind, wentalizing

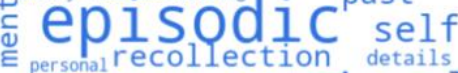

memory retrieval
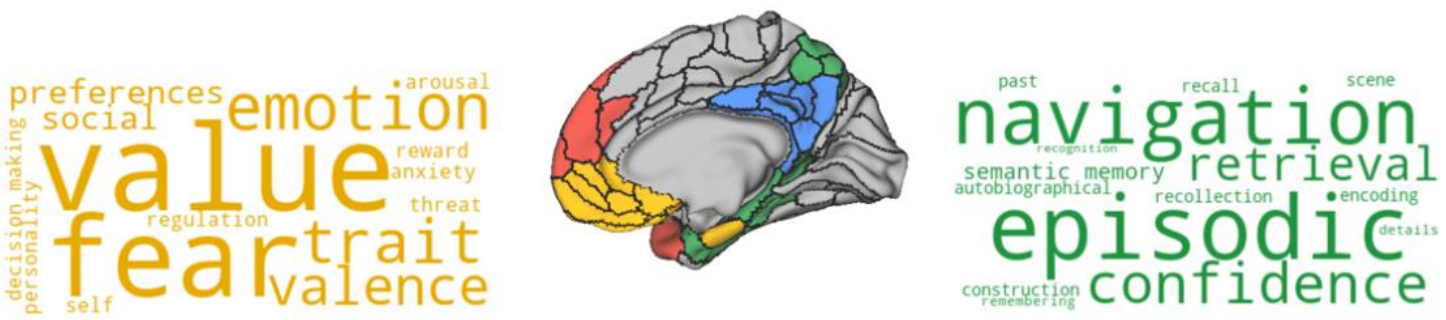

387 Figure 7. Terms associated with activation of each cortico-hippocampal network. Word clouds

388 are colored according to the network they describe which are displayed on the inflated brain. The

389 size of the terms in the word cloud relates to how strongly the meta-analytic activity of that term 
correlates with the spatial extent of the network. Red, AT subnetwork; Blue, PM subnetwork;

391 Yellow, MP subnetwork; Green, Medial temporal network.

\section{Discussion}

394 We have taken a data-driven approach using resting-state fMRI to identify and characterize

395 cortico-hippocampal network connectivity, finding a set of subnetworks that interact with the

396 hippocampus. Our analyses showed that the MTN, a cluster of regions in the medial temporal

397 and dorsomedial parietal lobes, could be differentiated from the DMN, which included medial

398 and lateral parietal, temporal, and frontal regions. This MTN mediates connectivity between the

399 visual network and the DMN and between the visual network and hippocampus. We also found

400 that the DMN could be meaningfully subdivided into three subnetworks: the PM subnetwork,

401 encompassing the posterior cingulate, lateral parietal, and dorsal lateral prefrontal cortex, the AT

402 subnetwork, encompassing the temporopolar, lateral orbitofrontal, and dorsal medial prefrontal

403 cortex, and the MP subnetwork, encompassing the ventral medial prefrontal, and entorhinal

404 cortex. These cortico-hippocampal subnetworks vary in connectivity strength along the long-axis

405 of the hippocampus, and meaningfully segregate based on feature representation as measured by

406 multivoxel activity patterns in an independent dataset.

407 Prior work on the neurobiology of memory has drawn on the idea that the hippocampal

408 formation primarily interacts with the PHC and PRC, such that these areas collectively comprise

409 a "MTL memory system" (2) that is functionally distinct from surrounding cortical areas. More

410 recently, we and others proposed that PHC and PRC are embedded in larger scale cortico-

411 hippocampal networks as evidenced by divergent anatomical pathways found in rodents and non-

412 human primates (19-22) and by differing functional connectivity of the PHC and PRC in humans 
$413(24,25,71)$. In this "PM/AT" framework, the PHC is a core region in the PM network, which

414 also includes retrosplenial, posterior cingulate and lateral parietal cortex, whereas the PRC is a

415 core region in the AT network, which also includes temporopolar, and orbitofrontal cortex (29).

416 As shown in Figure 8, the present results were not fully consistent with either the original PMAT

417 framework. We found that the PRC, PHC, retrosplenial cortex, inferior lateral parietal, and

418 dorsal medial parietal formed the MTN, and this network could be distinguished from the DMN.

419 This network corresponds closely to the "parieto-medial temporal pathway" described by Kravitz

420 et al. (72). This network division converges with previous data-driven community detection

421 studies $(15,42,47,73)$ showing that the MTN can be differentiated from the DMN and support

422 recent suggestions that heterogeneity exists within the PM system $(38,50)$. Critically, both the

423 MTN and DMN showed strong functional connectivity with the hippocampus.
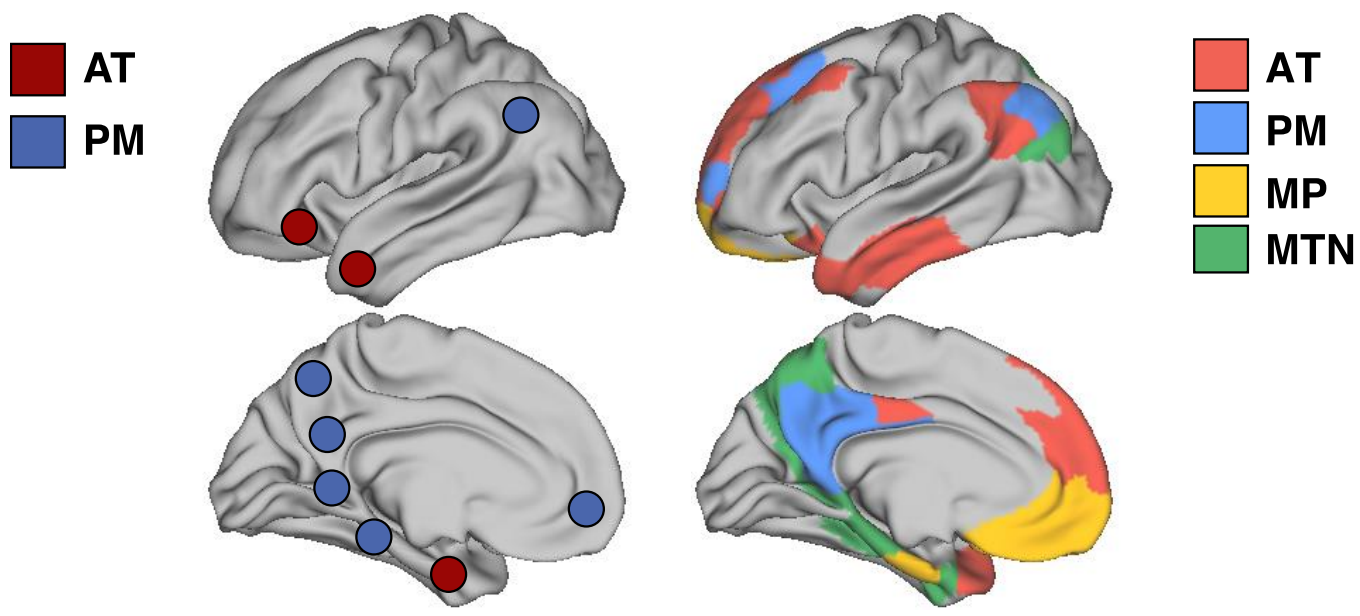

426 Figure 8. Left. Hypothesized PM/AT divisions with PM nodes as blue and AT nodes as red (29,

427 74). Right. Data-driven network assignments.

Although the present work shows that the MTN could be differentiated from the DMN, 
431 terms of connectivity. Prior anatomical studies in animal models $(75,76)$ and fMRI studies in

432 humans have shown that the PRC and PHC have different functional connectivity patterns (24-

433 26, 77). In fMRI studies, these differences are most apparent when the functional connectivity

434 maps of the PRC and PHC are directly contrasted to identify regions that show

435 disproportionately high connectivity to the PRC or the PHC. When not contrasted, the two

436 regions frequently show strong functional connectivity to each other and considerable overlap in

437 the spatial extent of their functional connectivity $(24,25,77)$. For example, Wang et al (25) and

438 Zhuo et al. (77) showed that the PHC and posterior PRC show high connectivity with each other,

439 and with medial parietal, medial prefrontal, lateral parietal and temporopolar regions. Therefore,

440 the evidence presented here should not be taken to reflect the presence or absence of functional

441 distinctions between PHC and PRC (as prior work has clearly shown that certain regions connect

442 more to PHC or PRC), but rather they demonstrate that when taking into account the topology of

443 functional connections across the entire neocortex, the MTN can be segregated from the DMN.

444 This evidence suggests that heterogeneity exists within the previously reported PM network.

445 Treating the brain as an abstract graph, we observed that the MTN serves as a critical

446 bridge between the visual network and the DMN and hippocampus, such that removal of the

447 MTN results in considerable loss of cross-network connectivity between the visual network and

448 DMN/hippocampus. The medial temporal cortex is thought to provide high-level feature

449 representation of the external world to the hippocampus which then binds those representations

450 in context $(4,22,29,58)$. Damage to the medial temporal cortex is often associated with varying

451 level of circumscribed memory impairment. Our results here suggest that loss of MTN

452 connections would result in disconnection between the external visual world and the

453 hippocampus and DMN, which could result in memory impairment. Using a novel analytic 
454 method, a recent study mapped the functional connectivity of regions in the brain where lesion

455 damage resulted in amnesia and found a common functional network to all the lesions (11). This

456 lesion functional connectivity map encompassed the DMN, MTN and encroached into visual

457 network regions as described in this study, underscoring the importance of these networks in

458 memory processing. Another study of amnesia following traumatic brain injury observed

459 reduced functional connectivity between the PHC and PCC was related to memory impairment

460 and normalized when memory functioning improved (78).

The distinction between the MTN and the DMN is consistent with recent studies

462 suggesting heterogeneity within the PM network $(14,38,50,79)$. Based on its network

463 properties, the MTN sits in a reasonable position to represent higher-order features of the visual

464 world to the DMN which is spatially distal from networks involved in primary sensation. Recent

465 theories of event cognition have suggested a separation between content (higher order

466 representations of current environment) and structure (abstracted knowledge pertaining the

467 typical way in which sequences of events unfold) (80). One possible instantiation of this

468 separation is that the MTN represents content of events whereas the DMN represents abstracted

469 structure. Indeed, a recent study has shown that when orienting to retrieval of perceptual features

470 during autobiographical recall, there is increased activity in the MTL, retrosplenial, and dorsal

471 precuneus (regions in the MTN) compared to when recall is oriented to conceptual or thematic

472 elements that preferentially resulted in activity of posterior cingulate, medial prefrontal, temporal

473 polar and lateral parietal cortex (regions in the DMN) (39). Another study found a similar

474 separation between the MTN and DMN by having participants encode a set of movie clips that

475 were considered typical (events that one would be familiar with through day to day life or

476 through movies/stories) or atypical (very unusual or dissimilar to anything you would encounter 
477 in day to day life or in movie/stories) (40). Regions in the DMN showed increased activity

478 during encoding of typical compared to atypical events and the reverse was true for regions in

479 the MTN. During encoding of typical events, activity in the DMN was interpreted as being

480 related to retrieval of relevant prior event knowledge to assist in comprehension and integration

481 of incoming information. Conversely, during encoding of atypical events, the activity in the

482 MTN was interpreted to reflect the need to encode the relations between items, contexts and

483 actions in the absence of a strong semantic framework. While these studies present strong

484 evidence to suggest a distinction between the MTN and DMN, it is important to note that in

485 many circumstances involving memory recall, these networks will be working in tandem to

486 support retrieval of both content and structure with some degree of bias that may favor retrieval

487 of item-level, scene-level or event-level elements. For example, one might expect that, even

488 within the MTN, a contrast that focusses on the distinction between different types of content,

489 such as scenes versus objects, may split regions within the MTN that have a bias towards those

490 content types (35). These findings demonstrate the necessity of our intrinsic connectivity

491 analyses to clarify that these differences in patterns of functional activity are likely due to the

492 presence of distinct functional networks.

493 The second major finding in the present study is that the DMN could be differentiated

494 into three subnetworks: the PM, AT, and MP subnetworks. The PM subnetwork consisted of

495 posterior cingulate, angular gyrus, and dorsal prefrontal cortex, whereas the AT subnetwork

496 consisted on temporopolar, lateral temporal, orbitofrontal and dorsal medial prefrontal cortex.

497 The collection of regions in the PM and AT subnetwork are in line with the PM/AT framework

$498(24,25)$, with the notable exception that the parahippocampal, retrosplenial cortex were not in

499 the PM subnetwork, and the perirhinal cortex was not in the AT subnetwork. The PM and AT 
500 subnetworks also resemble DMN subnetworks identified by previous data-driven methods (15,

501 41, 42), confirming that the PM and AT distinctions theorized do indeed persist, but they form

502 distinct communities from their MTN counterparts when examining their connectivity

503 architecture.

The MP subnetwork consists of medial prefrontal cortex, along with the entorhinal cortex

505 and was not explicitly predicted by prior theories. The medial prefrontal cortex has long been

506 distinguished from the orbitofrontal cortex based on anatomical connectivity $(21,22,81)$, and is

507 often grouped together with the posterior cingulate cortex as one of the "core hubs" of the DMN

$508(15,41,42)$. The entorhinal cortex certainly has dense interconnections with the medial

509 prefrontal cortex (81), but also is strongly interconnected with the hippocampus and adjacent

510 medial temporal cortex $(19,22,82)$. Interestingly, the entorhinal cortex often is part of the DMN

511 even when the MTL cortex is distinguished from the rest of the DMN $((73,83)$, but see $(15,47))$.

512 Furthermore, network analysis on histologically-defined axonal connections between cortical

513 regions in rodents, showed that the lateral entorhinal cortex was part of a community with the

514 prefrontal cortex, consistent with our partition, though the medial entorhinal cortex was placed in

515 a community with the hippocampus, retrosplenial cortex and subiculum (84). A distinction in the

516 functional connectivity of entorhinal cortex subregions has been shown in high-resolution human

517 resting state data $(23,37)$, but the spatial resolution of the current study is unable to resolve these

518 subregions and future work will be important to further characterize its connectivity in humans.

519 We examined the topology of how these subnetworks connect to each other and the rest

520 of the brain finding that the MP subnetwork had the highest within-DMN participation

521 coefficient, with nodes connecting broadly to AT and PM regions. Conversely, the MP

522 subnetwork had a low out of DMN participation coefficient, especially relative to the AT 
523 subnetwork. Our results provide converging evidence for a recent study published by Gordon et

524 al (85) that also found that a set of lateral networks resembling the AT subnetwork here, showed

525 the highest participation coefficient out of network. Their AT equivalent showed

526 intercommunication with language and frontoparietal networks, as was also seen here. The

527 participation coefficient findings suggest that the AT subnetwork and several high participation

528 PM nodes are positioned to mediate information transfer in and out of the DMN, whereas the MP

529 subnetwork may integrate and coordinate information within the DMN. A recent review of the

530 human lesion literature has reported that robust memory impairment can be observed following

531 medial prefrontal cortex damage and suggested that this may be due to the fact that the medial

532 prefrontal cortex has a role in initiating and coordinating cognitive processes for retrieval and

533 episodic simulation (86). Given the network findings here, and implications from the lesion

534 literature, the MP subnetwork is well positioned for higher-order integration and coordination of

535 memory-guided activity.

Differentiation of the three DMN subnetworks was reinforced by evidence from an

537 independent dataset in which we found that regions grouped in the same network represented

538 similar kinds of information, as compared to regions in different networks. These findings

539 replicate and extend results from previous studies that identified different functional differences

540 between different cortico-hippocampal networks $(17,69)$. For instance, Ritchey et al. (17) used

541 community detection to differentiate networks of regions hypothesized to show different patterns

542 of MTL connectivity. Ritchey et al. (17) interrogated these networks in an independent study of

543 episodic memory retrieval, as did Inhoff et al. (69) during a concept learning task. The functional

544 distinction of these networks was supported by findings showing that regions grouped in the

545 same network had greater representational profile similarity (17) and activation profile similarity 
546 than regions grouped in different networks during learning and memory tasks $(17,69)$. In these

547 past studies, the regions used in network construction were those that showed a significant

548 difference in functional connectivity between the PRC and PHC, being motivated by anatomical

549 evidence suggesting parallel pathways in the MTL (19). Whereas the seed selection that defined

550 these networks was based specifically on regions that had different functional connectivity

551 between PHC and PRC, in the current study, we did not impose any pre-existing assumptions,

552 and identified networks holistically in the context of the entire brain. We saw reliably higher

553 representational profile similarity for regions in the same network — a pattern consistently found

554 in every subject, highlighting the functional significance of these cortico-hippocampal networks

555 and validating our delineation of these cortico-hippocampal networks.

556 We also found that the MTN and DMN subnetworks show variation in their connectivity

557 across the anterior and posterior hippocampus. The hippocampus is known to vary along its long

558 axis in terms of intrinsic connectivity, extrinsic connectivity, receptor distribution, and gene

559 expression $(19,20,87-90)$. In humans, resting state functional connectivity has been the primary

560 tool for examining differences in connectivity along the hippocampal longitudinal axis, though

561 distinctions between the anterior and posterior hippocampus have been observed using DTI (28).

562 The anterior hippocampus has been shown to have greater connectivity to inferior temporal,

563 temporopolar, orbitofrontal, medial prefrontal and PRC, whereas the posterior hippocampus has

564 been shown to have greater connectivity to PHC, retrosplenial cortex, inferior parietal cortex,

565 precuneus, the anterior thalamus $(24,26-28,63)$. Here, we replicated and extended these

566 findings to characterize hippocampal connectivity to the networks formed by these cortical

567 regions. We found that the anterior hippocampus has relatively greater connectivity to the MP

568 and AT subnetworks, whereas the posterior hippocampus has relatively greater connectivity to 
569 the MTN. The preferential connectivity to the anterior hippocampus was relatively consistent

570 across all regions in the MP and AT subnetworks, but there was heterogeneity in the MTN's

571 preference for the anterior and posterior hippocampus with the anterior hippocampus having

572 stronger connectivity to anterior MTL cortex and the posterior hippocampus having relatively

573 stronger connectivity to dorsal medial parietal cortex. Theories regarding the functional

574 difference of the anterior and posterior hippocampus are often informed by preferential long-axis

575 connectivity to the rest of the brain $(16,91)$, but they do not consider how the rest of the brain

576 connects to form networks. The results presented here suggest the possibility that functional

577 differences between the anterior and posterior hippocampus may reflect differences in their

578 connections to the subnetworks of the DMN.

579 To better understand the functional specializations of these networks, we used meta-

580 analytic maps from the Neurosynth database to identify the cognitive constructs that have been

581 reliably associated with regions in the DMN subnetworks. Activity within the MTN was

582 associated with a set of terms heavily rooted in episodic memory and navigation. Activation in

583 the MP subnetwork, in contrast, was highly associated with terms pertaining to emotion, and

584 value. As noted above, the MTN (in particular the dorsal precuneus and dorsal posterior

585 cingulate) had greater connectivity to the posterior relative to the anterior hippocampus, whereas

586 the MP subnetwork had greater connectivity with the anterior compared to posterior

587 hippocampus. These connectivity patterns and neural decoding results converge with theories

588 linking the posterior hippocampus to cognitive and navigational processes and the anterior

589 hippocampus in motivation and emotional behavior (92). We also found that activation in the AT

590 subnetwork was associated with terms that relate to social cognition, theory of mind, and beliefs,

591 in line with contemporary theories (93) and lesion evidence (94) of this network. In the PM 
592 subnetwork, Neurosynth decoding revealed cognitive terms related to episodic memory and the

593 self. This is echoed by recent work, taking a highly subject-specific approach, that found a

594 distinction within the DMN between regions involved in episodic versus theory of mind-like

595 processes (95). In this study DiNicola et al (95) identified two distinct subnetworks of the DMN

596 on a subject-by-subject, voxel-wise basis. One subnetwork resembled the AT subnetwork and the

597 other resembled the PM subnetwork described here. The same subjects also performed both

598 episodic projection tasks and theory of mind tasks and it was observed that the voxels classified

599 as being in the AT-like network showed greater activity for the theory of mind tasks, whereas the

600 voxels classified as being in the PM-like network showed greater activity for the episodic

601 projection tasks. Interestingly, the Neurosynth analysis showed that the term "autobiographical"

602 was strongly associated with all four cortico-hippocampal networks. This broad association may

603 be due to the fact that autobiographical memory tasks usually involve retrieval of episodically

604 rich events that dynamically unfold involving social interactions, and relevance to the self (96).

605 Although the Neurosynth decoding analysis is exploratory, and by definition, post-hoc,

606 they can guide the generation of hypotheses to be tested in future studies. Further studies, similar

607 to the work of DiNicola et al (95), should examine whether MTN and DMN subnetworks

608 segregate based on spatial, valuation, and social processes, within the same group of individuals.

609 For example, during encoding and recall of complex events, we might expect regions within the

610 MTN to represent high-level object and context features, regions within the AT subnetwork to

611 represent interpretations of theory of mind and social interactions, and regions within the MP

612 subnetwork to represent the emotional valence or perceived value of the event. Indeed, a recent

613 preprint demonstrated a dissociation between the MTN and MP subnetwork during event

614 simulation, with increased activity in the MTN occurring when subjects were instructed to 
615 simulate past or future events using cues that were selected to elicit high levels of vividness,

616 whereas increased MP activity was observed when cues elicited simulations with high levels of

617 valence (97). Further, based on the functional connectivity of the MP subnetwork, we

618 hypothesize that it would be well suited to serve a coordinating role in network rearrangement to

619 accomplish task goals and demands. Again, a recent study by Nawa and Ando (98) found that

620 when elaborating on autobiographical memories, the vmPFC drove hippocampal activity and this

621 effect was augmented for memories that were of greater emotional valence. Further, damage to

622 the medial prefrontal cortex is associated with memory impairments characterized by difficulties

623 in elaborating on memories unfold over time and events (86). We hypothesize that these

624 impairments may be associated with dysfunction of network dynamics due to a loss of the

625 coordination normally provided by the MP subnetwork.

Finally, we note that recent studies have demonstrated the value in taking subject-specific

627 approaches to delineating functional networks in a voxel-wise fashion $(14,47)$. This work has

628 shown that there are individual differences in the location of boundaries between functional

629 networks, though it is notable that the majority of the cortex is labelled consistently in the

630 majority of participants (73). A key goal of the present study was to provide a clear framework to

631 guide analyses in future task-fMRI studies, in which it might not be feasible to obtain

632 independent, subject-specific parcellations. We have listed each region and its community label

633 in Supplementary Table 3, and the HCP-MMP atlas is available online

634 (https://balsa.wustl.edu/study/show/RVVG, (44)). Task fMRI studies that use group-level

635 analyses can use the group-level characterization of cortico-hippocampal networks reported here

636 in order to rigorously test hypotheses about the functions of these networks and how they may be

637 implicated in memory disorders, as resting connectivity has shown to predict spread of pathology 
638 in Alzheimer's (99), and DMN subnetwork perturbation has been demonstrated in MTL amnesia

639 (100).

640

641 Conclusions

642 The hippocampus affiliates with a broad set of regions that enable episodic retrieval. Here, we

643 have shown that the MTN and three subnetworks of the DMN can be differentiated on the basis

644 of their whole-brain functional connectivity. The three DMN subnetworks vary in connectivity

645 along the hippocampal long axis, have distinct representational roles during a memory task, and

646 topologically are connected by a set of hubs in the medial prefrontal subnetwork. This

647 subnetwork organization offers a novel framework to investigate event cognition and memory

648 retrieval.

650 Methods

$651 \quad \underline{\text { Participants }}$

652 Forty-five healthy, young adult participants were recruited from the University of California,

653 Davis and surrounding area $\left(\mathrm{N}_{\text {Females }}=26\right.$, mean age $=25.6$ years $[\mathrm{SD}=4.2$ years $\left.]\right)$. All

654 participants were right-handed and neurologically healthy. The study was approved by the

655 Institutional Review Board of the University of California at Davis and all participants provided

656 informed consent prior to participation. Participants were compensated \$20/hour for their time.

657 This sample size is comparable to the cohort sample sizes from the seminal Power et al. (101)

658 study investigating functional brain organization. There is no specific effect size that can be

659 taken to approximate power for identifying network communities, but previous studies have 
660 shown high reliability across subjects in network identification when using greater than 10

661 minutes of resting-state data $(73,102)$.

662

$663 \quad$ MRI Acquisition

664 MRI scanning was performed using a 3T Siemens Skyra scanner system with a 32-channel head

665 coil. A T1-weighted structural images was acquired using a magnetization prepared rapid

666 acquisition gradient-echo pulse sequence $\left(\mathrm{TR}=2100 \mathrm{~ms} ; \mathrm{TE}=2.98 \mathrm{~ms}\right.$; field of view $=256 \mathrm{~mm}^{2}$

667 ; flip angle $=7^{\circ}$; image matrix $=256 \times 256,208$ axial slices with $1.0 \mathrm{~mm}^{3}$ thickness; GRAPPA

668 acceleration factor 2 with 24 reference lines). Functional images were acquired using a gradient

669 EPI sequence $\left(\mathrm{TR}=1220 \mathrm{~ms} ; \mathrm{TE}=24 \mathrm{~ms} ;\right.$ field of view $=192 \mathrm{~mm}^{2} ;$ image matrix $=64 \times 64$;

670 flip angle $=67^{\circ} ;$ bandwidth $=2442 \mathrm{Hx} /$ pixel; multiband factor $=2 ; 38$ interleaved axial slices,

671 voxel size $=3 \mathrm{~mm}^{3}$ isotropic). Five runs of five minutes in duration were acquired for rest scans

672 for a total of 25 minutes of resting state fMRI data per subject. Participants were instructed to lay

673 as still as possible and try not to fall asleep.

\section{Anatomical Preprocessing}

676 T1-weighted anatomical scans were preprocessed using FreeSurfer which included intensity

677 normalization, removal of non-brain tissue, transformation to Talaraich space, and segmentation

678 of gray matter, white matter, and CSF. Surfaces were calculated for the white matter-gray matter,

679 and gray matter-pial interface. Surface-based registration to the HCP-MMP1.0 atlas (44) was

680 performed and subject-specific cortical regions were calculated according to atlas boundaries.

681 Surface-based cortical regions were converted to volumetric regions of interest and transformed

682 into functional native space. The hippocampus was segmented in FreeSurfer in an automated 
683 fashion. Manual adjustments were done to correct misclassified voxels and the hippocampus was

684 divided into anterior and posterior segments based off of the disappearance of the uncal notch

685 (16), with the posterior hippocampus designated as all of the hippocampus posterior to the

686 disappearance of the uncal notch on a coronal slice.

$688 \quad$ Functional MRI Preprocessing

689 Functional preprocessing was performed using fMRIPrep 1.4.1 ((103, 104),

690 RRID:SCR_016216), which is based on Nipype 1.2.0 ((105, 106), RRID:SCR_002502). For each

691 of the 5 BOLD runs found per subject (across all tasks and sessions), the following preprocessing

692 was performed. First, a reference volume and its skull-stripped version were generated using a

693 custom methodology of fMRIPrep. A deformation field to correct for susceptibility distortions

694 was estimated based on two echo-planar imaging (EPI) references with opposing phase-encoding

695 directions, using 3dQwarp from Cox and Hyde (107) (AFNI 20160207). Based on the estimated

696 susceptibility distortion, an unwarped BOLD reference was calculated for a more accurate co-

697 registration with the anatomical reference. The BOLD reference was then co-registered to the

698 T1w reference using flirt (FSL 5.0.9, (108)) with the boundary-based registration (109) cost-

699 function. Co-registration was configured with nine degrees of freedom to account for distortions

700 remaining in the BOLD reference. Head-motion parameters with respect to the BOLD reference

701 (transformation matrices, and six corresponding rotation and translation parameters) are

702 estimated before any spatiotemporal filtering using mcflirt (FSL 5.0.9, (110)). The BOLD time-

703 series were resampled onto their original, native space by applying a single, composite transform

704 to correct for head-motion and susceptibility distortions. These resampled BOLD time-series will

705 be referred to as preprocessed BOLD. Several confounding time-series were calculated based on 
the preprocessed BOLD as part of fMRIPrep, however, only the head-motion estimates

calculated in the correction step and frame displacement (FD) were used in subsequent analyses.

All resamplings can be performed with a single interpolation step by composing all the pertinent

transformations (i.e. head-motion transform matrices, susceptibility distortion correction when

710 available, and co-registrations to anatomical space). Gridded (volumetric) resamplings were

711 performed using antsApplyTransforms (ANTs), configured with Lanczos interpolation to

712 minimize the smoothing effects of other kernels (111). Many internal operations

713 of fMRIPrep use Nilearn 0.5.2 ((112), RRID:SCR_001362), mostly within the functional

714 processing workflow. For more details of the pipeline, see

715 https://fmriprep.readthedocs.io/en/stable/workflows.html.

716 The preprocessed BOLD timeseries, anatomical images, and native space Glasser parcels

717 were imported into CONN Toolbox version 18b (www.nitrc.org/projects/conn,

718 RRID:SCR_009550). Based on Ciric et al.'s (113) assessment of 14 common denoising

719 protocols, we selected the '9p' protocol because it was shown to facilitate the highest functional

720 network identifiability (see (113) Figure 5b). BOLD timeseries were demeaned, linear and

721 quadratic trends were removed, and bandpass filtered between .008 and $.09 \mathrm{hz}$. The 9p protocol

722 includes as confound regressors six motion parameters, WM, CSF, and global signal regression.

723 All regressors were band-pass filtered to maintain the same frequency range as the data. Visual

724 inspection of the frequency distributions of functional connectivity values as well as quality

725 control plots of the correlation between mean FD and functional connectivity values was

726 performed to identify any aberrant runs or subjects. Five outlier subjects were identified which

727 each corresponded to previously identified subjects having a mean FD value of greater than

$728.15 \mathrm{~mm}$. These subjects were excluded from further analyses. Functional connectivity matrices 
were created for each subject by computing Pearson's correlations between all possible pairs of

730 each regions' confound-corrected timeseries. Finally, each correlation value was Fisher z-

731 transformed with the inverse hyperbolic tangent function.

\section{Community Detection}

734 Cortical functional connectivity (FC) matrices from all subjects were thresholded to exclude

735 negative connections that may be introduced by global signal regression $(114,115)$ and then

736 averaged together. Using the resulting group averaged connectivity matrix, community detection

737 was performed using the Louvain method (45) via the brain connectivity toolbox

738 (https://sites.google.com/site/bctnet/) which iteratively performs a greedy optimization of

739 modularity by randomly selecting nodes and merging them into the community that maximally

740 increases modularity, until no more gains in modularity are possible. Modularity (Q) describes

741 how well a network can be divided into communities that have higher within-community

742 connectivity than would be expected by chance and can range from -1 to 1 , with negative values

743 indicating fewer intracommunity connections than would be expected by chance and positive

744 values indicating higher intracommunity connections than would be expected by chance (56).

745 We used a method of the Louvain community detection algorithm adapted to accommodate

746 weighted graphs (116) which accepts the weighted, group average functional connectivity

747 matrix. This algorithm can be tuned using a resolution parameter, gamma, that biases the

748 algorithm towards producing few, large networks (low gamma) or towards many, smaller

749 networks (high gamma). To determine the resolution parameter in a principled manner, we

750 adopted the approach used by Ji et al. (43). The criteria included: i) separation of the primary

751 sensory-motor networks (visual, auditory, somatomotor) from all other networks, ii) high 
752 stability across nearby parameters (similar network partitions across neighboring parameter

753 settings), and iii) optimized modularity.

754 Given that the Louvain algorithm detection is influenced by a random starting point, for

755 every tested resolution parameter, we ran the algorithm 1000 times. To determine the optimal

756 partitioning solution at each resolution, gamma, we examined how consistent a given partitioning

757 solution was to every other solution produced over the 1000 iterations at the same resolution.

758 Consistency was calculated using the z-Rand score (46) (http://commdetect.weebly.com/). For

759 each partition solution, we weighted the mean z-Rand score (consistency) of the partition with

760 the modularity value of that partition to select a partition solution that was both highly consistent

761 at a given resolution and had high modularity. These methods are in keeping with Ji et al. (43).

762 Using the default parameter, gamma $=1$, we could not satisfy criteria i) as the somatomotor and

763 auditory network were grouped together (also seen in Ji et al. (43)). Thus, we ran a parameter

764 sweep from gamma $1-2.8$ by increments of 0.005 until we identified a resolution parameter at

765 which the somatomotor and auditory network separated consistently and produced the highest

766 modularity-weighted z-Rand score.

768 Path Length Analysis

769 Using the brain connectivity toolbox (https://sites.google.com/site/bctnet/), weighted path length

770 was calculated between all nodes in the brain for each subject, after excluding negative

771 connectivity weights. Path length is the fewest number of links that need to be traversed to

772 connect two nodes. To calculate the weighted path length, the functional connectivity weights

773 were inverted creating a distance matrix in which nodes with high functional connectivity had

774 low distance. Then, the shortest path between each pair of nodes was calculated providing a 
775 value that represented the minimal the total weighted distance that needed to be traversed to

776 connect two nodes.

777 We hypothesized that removal of the MTN would reduce the ability of the visual network 778 and DMN/hippocampus to connect with each other. To test this hypothesis, all the nodes from

779 the MTN were removed from each subject's network and the average weighted path length was

780 calculated between visual network and DMN and between visual network and hippocampus.

781 Because removal of nodes can only lead to an increase in path length (shorter paths can be

782 removed, but will never be added following removal of nodes), we compared the weighted path

783 length following removal of the MTN to the weighted path length following removal of every

784 other network, using paired t-tests. As a secondary control, we also compared the weighted path

785 length following removal of the MTN to the distribution of weighted path lengths following

786 removal of 30 random nodes (other than the DMN, visual network and hippocampus) over 1000

787 iterations. For this, we calculated the z-score of the weighted path length following removal of

788 the MTN compared to the distribution of weighted path lengths following removal of 30 nodes

789 over 1000 iterations. This was done for each subject such that each subject had a corresponding

790 z-score. A one-sample t-test was then performed on the z-score values to examine whether they

791 were significantly greater than 0 .

792 Community Partitioning of the Default Mode Network

793 From the identified whole brain partitioning, we selected the default mode network based on

794 visual similarity to previously identified DMN solutions $(42,43,117)$. We repeated the

795 community detection procedure described above on the identified DMN (1000 iterations of

796 Louvain community detection at each resolution parameter) and calculated modularity-weighted 
z-rand scores across a range of resolution parameters, $0.75-1.1$ at increments of 0.05 . We

798 selected local modularity-weighted z-rand peaks as solutions for further exploration.

800 Connection Diversity in the Default Mode Network

801 The participation coefficient, which quantifies the degree to which a node is connected to a

802 diverse set of communities, was calculated using the tools from the brain connectivity toolbox

803 (https://sites.google.com/site/bctnet/). Since the participation coefficient calculation uses node

804 strength (the sum of the connectivity weights across the network) in the denominator of its

805 calculation, nodes with unusually low strength can produce unstably large participation

806 coefficient values. We, thus, excluded regions with node strength in the bottom $25 \%$ of the

807 network hubs. Participation coefficient calculations were performed across a range of network

808 densities from 5-20\% at $1 \%$ intervals. These methods for calculating participation coefficient are

809 in line with best practices in the literature $(57,59,62,118,119)$. Whole network participation

810 coefficient was calculated for each DMN region, using the whole brain connectivity matrix and

811 community labels generated from the whole-brain Louvain community detection. Within DMN

812 participation coefficient was also calculated for each DMN node, using only the connections

813 within the DMN and using the DMN subnetwork labels. Participation coefficient values were

814 compared between DMN subnetworks using a within-subjects mixed model using mixedlm from

815 statsmodels in Python 3 (120).

817 Hippocampal-cortical network connectivity

818 To identify hippocampal-cortical networks we calculated functional connectivity between each

819 hippocampal ROI and every cortical ROI in the brain. To identify average connectivity of the 
820 hippocampus to a given network within-subject, functional connectivity weights were averaged

821 together across cortical nodes, based on community affiliation. A one-sample t-test was

822 performed to determine what networks were, on average, significantly connected to the

823 hippocampus. Significance was set at $p<.05$, Bonferroni corrected. We examined the

824 connectivity of the resulting significant networks and subnetworks to the hippocampus as a

825 function of the long-axis (anterior versus posterior) and hemisphere (left versus right) in a

826 within-subjects model as described above.

827 ROI level functional connectivity difference between the anterior and posterior

828 hippocampus were performed in CONN toolbox version $18 \mathrm{~b}$. Using the cortical ROIs that were a

829 part of large-scale networks connected to the hippocampus, a within-subjects analysis was

830 performed which contrasted functional connectivity in the left and right anterior hippocampus

831 against the left and right posterior hippocampus. Significance was set at $p<.05, F W E$ corrected.

833 Representational Similarity Analysis

834 Using an independent dataset collected during a memory-based decision-making task (68), we

835 examined the representational profile of each ROI in the DMN subnetworks (See SI Appendix

836 for a detailed description of the task). If the hippocampal-cortical networks identified using

837 resting-state functional connectivity are functionally meaningful, then regions within those

838 networks should have similar representational profiles.

839 In brief, 22 participants in this dataset viewed a set of 8 grocery items one at a time, in a

840 pre-scanning session, and learned through trial and error whether each grocery item was

841 desirable to a hypothetical customer within a store context or not. In each trial, a grocery item

842 appeared in the context of a grocery store. There were four different grocery stores and for half 
843 of the items the particular grocery store modulated their desirability and for the other half, the

844 grocery store context was irrelevant to their desirability. For example, the apple at grocery store

845 'A' may be desirable, but the apple at grocery store 'B' may be undesirable which makes apple's

846 desirability context-dependent. Alternatively, desirability of a carton of milk may be the same

847 regardless or grocery store which makes it context-invariant. Overall preference averaged to $50 \%$

848 desirable for both context dependent and invariant. Further, of the four contexts, 50\% shared the

849 same preference rules. Following successful learning, participants were put in the scanner and

850 shown the context and a food item, one at a time and asked to remember whether the food item

851 was desirable to the hypothetical customer at the store context based on their learning phase.

852 Feedback was not provided during scanning. In the original study, Mizrak et al. (68) examined

853 representational similarity between memory-based decisions depending on a) shared features

854 between grocery items such as being modulated by the context or not, b) shared features between

855 store contexts such as having similar desirability for the same grocery items or not.

856 Here, using the RSA toolbox (https://www.mrc-cbu.cam.ac.uk/methods-and-

857 resources/toolboxes/license/, (121)), pattern similarity was calculated between memory based

858 decision making trials for each ROI using Pearson's correlation, excluding a) trials that occurred

859 during the same scanning run, and b) incorrect trials. Thus, fluctuations in pattern similarity

860 would be driven by variations in shared features. Correlations were then calculated between

861 ROIs to calculate the similarity of their representational profiles. We then examined the

862 representational similarity within cortico-hippocampal subnetworks versus between using a

863 paired t-test to examine whether these networks identified using resting-state functional

864 connectivity did indeed create functionally relevant communities. 
To account for univariate effects on the multivariate analysis, we further calculated the mean BOLD activity within an ROI for each trial and correlated the trial-wise mean BOLD

867 activity for each pair of ROIs to create an ROI-by-ROI activation similarity matrix. Using the

868 activation similarity matrix, we regressed out the influence of univariate activity from the RDM

869 similarity matrix and re-ran the statistical analysis contrasting within- vs. between-network RDM

870 similarity.

871 To account for the effects of spatial proximity between ROIs, we calculated the

872 Euclidean distance between the centroids of each pair of ROIs to create an ROI-by-ROI distance

873 matrix. Using the distance matrix, we regressed out the influence of spatial proximity from the

874 RDM similarity matrix and re-ran the statistical analysis contrasting within- vs. between-network

875 RDM similarity.

877 Cognitive characterization of hippocampal-cortical networks

878 We decoded cortico-hippocampal networks to determine what terms are most frequently

879 descriptive of the spatial distribution of the networks using the repository data version 0.7

880 (https://github.com/neurosynth/neurosynth-data) of Neurosynth

881 (https://github.com/neurosynth/neurosynth) (70). The meta-analytic activation of every term in

882 the Neurosynth database was correlated with the binarized volumetric masks of the hippocampal-

883 cortical networks. For each hippocampal-cortical network we examined the top terms whose

884 meta-analytic activation correlated most strongly with the spatial layout of the hippocampal-

885 cortical network. We removed terms that related to anatomy (e.g. ventromedial prefrontal

886 cortex), network (e.g. default mode), technique (e.g. independent component) and report terms

887 associated with cognitive functioning (e.g. navigation). We also removed terms that were 
888 redundant with other terms (e.g. "autobiographic" and "autobiographical memory"). When

889 removing redundant terms, the term that was more specific was retained (e.g. "autobiographical

890 memory" retained in favor of "memory"), otherwise the term with the higher correlation to the

891 network was retained. Word cloud visualizations were made using wordcloud 1.7.0

892 (https://pypi.org/project/wordcloud/).

893 


\section{References}

896 1. E. Tulving, Memory and consciousness. Can. Psychol. 26, 1-12 (1985).

897 2. L. R. Squire, S. Zola-Morgan, The medial temporal lobe memory system. Science 253,

$898 \quad 1380-6(1991)$.

899 3. H. Eichenbaum, A. P. Yonelinas, C. Ranganath, The medial temporal lobe and recognition

900 memory. Annu. Rev. Neurosci. 30, 123-52 (2007).

901 4. L. Davachi, Item, context and relational episodic encoding in humans. Curr. Opin.

$902 \quad$ Neurobiol. 16, 693-700 (2006).

903 5. T. J. Teyler, P. DiScenna, The Hippocampal Memory Indexing Theory. Behav. Neurosci.

$904 \quad$ 100, 147-154 (1986).

905 6. M. D. Rugg, K. L. Vilberg, Brain networks underlying episodic memory retrieval. Curr.

$906 \quad$ Opin. Neurobiol. 23, 255-260 (2013).

907 7. C. McCormick, M. Moscovitch, A. B. Protzner, C. G. Huber, M. P. McAndrews,

908 Hippocampal-neocortical networks differ during encoding and retrieval of relational

909 memory: Functional and effective connectivity analyses. Neuropsychologia 48, 3272-

$910 \quad 3281(2010)$

911 8. C. Sestieri, M. Corbetta, G. L. Romani, G. L. Shulman, Episodic memory retrieval,

912 parietal cortex, and the default mode network: Functional and topographic analyses. $J$.

$913 \quad$ Neurosci. 31, 4407-4420 (2011).

914 9. R. A. Cooper, M. Ritchey, Cortico-hippocampal network connections support the 915 multidimensional quality of episodic memory. Elife 8, 1-22 (2019).

916 10. B. Bellana, Z. X. Liu, N. B. Diamond, C. L. Grady, M. Moscovitch, Similarities and 
differences in the default mode network across rest, retrieval, and future imagining. Hum.

919 11. M. A. Ferguson, et al., A human memory circuit derived from brain lesions causing

920 amnesia. Nat. Commun. 10, 1-9 (2019).

921 12. M. D. Greicius, B. Krasnow, A. L. Reiss, V. Menon, Functional connectivity in the resting

922 brain: A network analysis of the default mode hypothesis. Proc. Natl. Acad. Sci. U. S. A.

$923 \quad \mathbf{1 0 0}, 253-258(2003)$.

924 13. J. R. Andrews-Hanna, J. Smallwood, R. N. Spreng, The default network and self-

925 generated thought: Component processes, dynamic control, and clinical relevance. Ann. N.

$926 \quad$ Y. Acad. Sci. 1316 (2014).

927 14. R. M. Braga, K. R. A. Van Dijk, J. R. Polimeni, M. C. Eldaief, R. L. Buckner, Parallel

928 distributed networks resolved at high resolution reveal close juxtaposition of distinct

929 regions. J. Neurophysiol. 121, 1513-1534 (2019).

930 15. T. J. Akiki, C. G. Abdallah, Determining the Hierarchical Architecture of the Human

931 Brain Using Subject-Level Clustering of Functional Networks. Sci. Rep. 9, 19290 (2019).

932 16. J. Poppenk, H. R. Evensmoen, M. Moscovitch, L. Nadel, Long-axis specialization of the

933 human hippocampus. Trends Cogn. Sci. 17, 230-40 (2013).

934 17. M. Ritchey, A. P. Yonelinas, C. Ranganath, Functional connectivity relationships predict

935 similarities in task activation and pattern information during associative memory

936 encoding. J. Cogn. Neurosci. 26, 1085-99 (2014).

937 18. M. J. Sekeres, G. Winocur, M. Moscovitch, The hippocampus and related neocortical

938 structures in memory transformation. Neurosci. Lett. 680, 39-53 (2018).

939 19. R. D. Burwell, The parahippocampal region: corticocortical connectivity. Ann. N. Y. Acad. 
Sci. 911, 25-42 (2000).

941 20. B. A. Strange, M. P. Witter, E. S. Lein, E. I. Moser, Functional organization of the

942 hippocampal longitudinal axis. Nat. Rev. Neurosci. 15, 655-669 (2014).

943 21. H. Kondo, K. S. Saleem, J. L. Price, Differential connections of the perirhinal and

944 parahippocampal cortex with the orbital and medial prefrontal networks in macaque

945 monkeys. J. Comp. Neurol. 493, 479-509 (2005).

946 22. J. P. Aggleton, Multiple anatomical systems embedded within the primate medial

947 temporal lobe: Implications for hippocampal function. Neurosci. Biobehav. Rev. 36,

$948 \quad$ 1579-1596 (2012).

949 23. A. Maass, D. Berron, L. A. Libby, C. Ranganath, E. Düzel, Functional subregions of the 950 human entorhinal cortex. Elife 4, 1-20 (2015).

951 24. L. A. Libby, A. D. Ekstrom, J. D. Ragland, C. Ranganath, Differential Connectivity of

952 Perirhinal and Parahippocampal Cortices within Human Hippocampal Subregions

953 Revealed by High-Resolution Functional Imaging. J. Neurosci. 32, 6550-6560 (2012).

954 25. S. Wang, M. Ritchey, L. a Libby, C. Ranganath, Functional connectivity based

955 parcellation of the human medial temporal lobe. Neurobiol. Learn. Mem. 134, 123-134

956 (2016).

957 26. I. Kahn, J. R. Andrews-Hanna, J. L. Vincent, A. Z. Snyder, R. L. Buckner, Distinct

958 cortical anatomy linked to subregions of the medial temporal lobe revealed by intrinsic

959 functional connectivity. J. Neurophysiol. 100, 129-39 (2008).

960 27. J. Poppenk, M. Moscovitch, A hippocampal marker of recollection memory ability among

961 healthy young adults: contributions of posterior and anterior segments. Neuron 72, 931-7

$962 \quad$ (2011). 
963 28. A. Adnan, et al., Distinct hippocampal functional networks revealed by tractography-

964 based parcellation. Brain Struct. Funct. 221, 2999-3012 (2016).

965 29. C. Ranganath, M. Ritchey, Two cortical systems for memory-guided behaviour. Nat. Rev.

$966 \quad$ Neurosci. 13, 713-726 (2012).

967 30. L. A. Libby, Z. M. Reagh, N. R. Bouffard, J. D. Ragland, C. Ranganath, The

968 Hippocampus Generalizes across Memories that Share Item and Context Information. $J$.

969 Cogn. Neurosci. 31, 24-35 (2019).

970 31. M. Ritchey, M. E. Montchal, A. P. Yonelinas, C. Ranganath, Delay-dependent

971 contributions of medial temporal lobe regions to episodic memory retrieval. Elife 2015, 1-

$97219(2015)$.

973 32. L. T. Hsieh, M. J. Gruber, L. J. Jenkins, C. Ranganath, Hippocampal Activity Patterns

974 Carry Information about Objects in Temporal Context. Neuron 81, 1165-1178 (2014).

975 33. S. Kim, et al., Selective and coherent activity increases due to stimulation indicate

976 functional distinctions between episodic memory networks. Sci. Adv. 4, 1-10 (2018).

977 34. K. N. Warren, M. S. Hermiller, A. S. Nilakantan, J. L. Voss, Stimulating the Hippocampal

978 posteriormedial network enhances task-dependent connectivity and memory. Elife 8, 1-21

979 (2019).

980 35. A. Maass, et al., Alzheimer's pathology targets distinct memory networks in the ageing $981 \quad$ brain. Brain 142, 2492-2509 (2019).

982 36. D. Berron, D. van Westen, R. Ossenkoppele, O. Strandberg, O. Hansson, Medial temporal 983 lobe connectivity and its associations with cognition in early Alzheimer's disease. Brain

$984 \quad \mathbf{1 4 3}, 1233-1248(2020)$.

985 37. J. N. Adams, A. Maass, T. M. Harrison, S. L. Baker, W. J. Jagust, Cortical tau deposition 
follows patterns of entorhinal functional connectivity in aging. Elife 8, 1-22 (2019).

987 38. M. Ritchey, R. A. Cooper, Deconstructing the Posterior Medial Episodic Network. Trends Cogn. Sci., 1-15 (2020).

989 39. L. Gurguryan, S. Sheldon, Retrieval orientation alters neural activity during autobiographical memory recollection. Neuroimage 199, 534-544 (2019).

991 40. K. Bonasia, et al., Prior knowledge modulates the neural substrates of encoding and retrieving naturalistic events at short and long delays. Neurobiol. Learn. Mem. 153, 26-39 (2018).

994 41. J. R. Andrews-Hanna, J. S. Reidler, J. Sepulcre, R. Poulin, R. L. Buckner, FunctionalAnatomic Fractionation of the Brain's Default Network. Neuron 65, 550-562 (2010).

996 42. B. T. T. Yeo, et al., The organization of the human cerebral cortex estimated by intrinsic functional connectivity. J. Neurophysiol. 106, 1125-1165 (2011).

998 43. J. L. Ji, et al., Mapping the human brain's cortical-subcortical functional network organization. Neuroimage 185, 35-57 (2019).

1000 44. M. F. Glasser, et al., A multi-modal parcellation of human cerebral cortex. Nature 108, $1001 \quad 125-38(2016)$.

1002 45. V. D. Blondel, J. L. Guillaume, R. Lambiotte, E. Lefebvre, Fast unfolding of communities 1003 in large networks. J. Stat. Mech. Theory Exp. 2008 (2008).

1004 46. A. L. Traud, E. D. Kelsic, P. J. Mucha, M. A. Porter, Comparing community structure to 1005 characteristics in online collegiate social networks. SIAM Rev. 53, 526-543 (2011).

1006 47. E. M. Gordon, et al., Precision Functional Mapping of Individual Human Brains. Neuron $1007 \quad 95,791-807 . e 7(2017)$.

1008 48. M. W. Cole, D. S. Bassett, J. D. Power, T. S. Braver, S. E. Petersen, Intrinsic and task- 
evoked network architectures of the human brain. Neuron 83, 238-251 (2014).

1010 49. A. W. Gilmore, S. M. Nelson, K. B. McDermott, The Contextual Association Network (2016).

1013 50. E. H. Silson, A. Steel, A. Kidder, A. W. Gilmore, C. I. Baker, Distinct subdivisions of 1014 human medial parietal cortex support recollection of people and places. Elife $\mathbf{8}, 554915$ $1015 \quad$ (2019).

1016 51. M. Peer, R. Salomon, I. Goldberg, O. Blanke, S. Arzy, Brain system for mental orientation 1017 in space, time, and person. Proc. Natl. Acad. Sci. U. S. A. 112, 11072-11077 (2015).

1018 52. M. Jacomy, T. Venturini, S. Heymann, M. Bastian, ForceAtlas2, a continuous graph 1019 layout algorithm for handy network visualization designed for the Gephi software. PLoS $1020 \quad$ One 9, 1-12 (2014).

1021 53. B. Fischl, et al., Whole brain segmentation: automated labeling of neuroanatomical 1022 structures in the human brain. Neuron 33, 341-55 (2002).

1023 54. J. L. Vincent, et al., Coherent Spontaneous Activity Identifies a Hippocampal-Parietal Memory Network. J. Neurophysiol. 96, 3517-3531 (2006).

1025 55. C. Baldassano, et al., Discovering Event Structure in Continuous Narrative Perception and 1026 Memory. Neuron 95, 709-721.e5 (2017).

1027 56. M. Rubinov, O. Sporns, Complex network measures of brain connectivity: Uses and 1028 interpretations. Neuroimage 52, 1059-1069 (2010).

1029 57. E. M. Gordon, et al., Three Distinct Sets of Connector Hubs Integrate Human Brain Function. Cell Rep. 24, 1687-1695.e4 (2018).

1031 58. R. A. Diana, A. P. Yonelinas, C. Ranganath, Imaging recollection and familiarity in the 
medial temporal lobe: a three-component model. Trends Cogn. Sci. 11, 379-386 (2007).

1033

1034

1035

1036

1037

1038

1039

1040

1041

1042

1043

1044

1045

1046

1047

1048

1049

1050

1051

1052

1053

1054

59. C. J. Lynch, et al., Precision Inhibitory Stimulation of Individual-Specific Cortical Hubs Disrupts Information Processing in Humans. Cereb. Cortex 29, 3912-3921 (2019).

60. D. E. Warren, et al., Network measures predict neuropsychological outcome after brain injury. Proc. Natl. Acad. Sci. U. S. A. 111, 14247-14252 (2014).

61. C. Gratton, E. M. Nomura, F. Pérez, M. D’Esposito, Focal brain lesions to critical locations cause widespread disruption of the modular organization of the brain. J. Cogn. Neurosci. 24, 1275-1285 (2012).

62. M. A. Bertolero, B. T. T. Yeo, M. D’Esposito, The diverse club. Nat. Commun. 8, 1-10 (2017).

63. L. E. Frank, C. R. Bowman, D. Zeithamova, Differential Functional Connectivity along the Long Axis of the Hippocampus Aligns with Differential Role in Memory Specificity and Generalization. J. Cogn. Neurosci. 31, 1958-1975 (2019).

64. I. Przeździk, M. Faber, G. Fernández, C. F. Beckmann, K. V. Haak, The functional organisation of the hippocampus along its long axis is gradual and predicts recollection. Cortex 119, 324-335 (2019).

65. M. P. McAndrews, M. Cohn, Neuropsychology in Temporal Lobe Epilepsy: Influences from Cognitive Neuroscience and Functional Neuroimaging. Epilepsy Res. Treat. 2012, $1-13$ (2012).

66. J. Miller, et al., Lateralized hippocampal oscillations underlie distinct aspects of human spatial memory and navigation. Nat. Commun. 9 (2018).

67. K. Iglói, C. F. Doeller, A. Berthoz, L. Rondi-Reig, N. Burgess, Lateralized human hippocampal activity predicts navigation based on sequence or place memory. Proc. Natl. 
Acad. Sci. U. S. A. 107, 14466-14471 (2010).

1056 68. E. Mizrak, N. R. Bouffard, L. A. Libby, E. Boorman, C. Ranganath, Representation of

1057 Task Structure in Human Hippocampus. bioRxiv, 794305 (2019).

1058 69. M. Inhoff, L. Libby, T. Noguchi, B. Love, C. Ranganath, Dynamic integration of conceptual information during learning. PLoS One 13, 1-26 (2018).

1060 70. T. Yarkoni, R. A. Poldrack, T. E. Nichols, D. C. Van Essen, T. D. Wager, Large-scale 1061 automated synthesis of human functional neuroimaging data. 8 (2011).

1062 71. I. Kahn, D. Shohamy, Intrinsic connectivity between the hippocampus, nucleus

1063 accumbens, and ventral tegmental area in humans. Hippocampus 23, 187-192 (2013).

1064 72. D. J. Kravitz, K. S. Saleem, C. I. Baker, M. Mishkin, A new neural framework for 1065 visuospatial processing. Nat. Rev. Neurosci. 12, 217-230 (2011).

1066 73. E. M. Gordon, T. O. Laumann, B. Adeyemo, S. E. Petersen, Individual Variability of the 1067 System-Level Organization of the Human Brain. Cereb. Cortex 27, 386-399 (2017).

1068 74. Z. M. Reagh, C. Ranganath, What does the functional organization of cortico-

1069 hippocampal networks tell us about the functional organization of memory? Neurosci.

$1070 \quad$ Lett. 680, 69-76 (2018).

1071 75. W. L. Suzuki, D. G. Amaral, Perirhinal and parahippocampal cortices of the macaque 1072 monkey: Cortical afferents. J. Comp. Neurol. 350, 497-533 (1994).

1073 76. P. Lavenex, W. A. Suzuki, D. G. Amaral, Perirhinal and parahippocampal cortices of the 1074 macaque monkey: Projections to the neocortex. J. Comp. Neurol. 447, 394-420 (2002).

1075 77. J. Zhuo, et al., Connectivity profiles reveal a transition subarea in the parahippocampal 1076 region that integrates the anterior temporal-posterior medial systems. J. Neurosci. 36, $1077 \quad 2782-2795(2016)$. 
1078 78. S. De Simoni, et al., Disconnection between the default mode network and medial

1079

1080

1081

1082

1083

1084

1085

1086

1087

1088

1089

1090

1091

1092

1093

1094

1095

1096

1097

1098

1099

1100

temporal lobes in post-traumatic amnesia Keywords: post-traumatic amnesia; traumatic brain injury; functional connectivity; default mode network; memory Abbreviations:

DMN = default mode network; M. Brain 139, 3137-3150 (2016).

79. E. R. Chrastil, S. M. Tobyne, R. K. Nauer, A. E. Chang, C. E. Stern, Converging metaanalytic and connectomic evidence for functional subregions within the human retrosplenial region. Behav. Neurosci. 132, 339-355 (2018).

80. S. M. Frankland, J. D. Greene, Concepts and Compositionality: In Search of the Brain's Language of Thought. Annu. Rev. Psychol. 71, 273-303 (2020).

81. S. T. Carmichael, J. L. Price, Sensory and premotor connections of the orbital and medial prefrontal cortex of macaque monkeys. J. Comp. Neurol. 363, 642-664 (1995).

82. W. A. Suzuki, D. G. Amaral, Topographic organization of the reciprocal connections between the monkey entorhinal cortex and the perirhinal and parahippocampal cortices. $J$. Neurosci. 14, 1856-1877 (1994).

83. S. Marek, et al., Identifying reproducible individual differences in childhood functional brain networks: An ABCD study. Dev. Cogn. Neurosci. 40 (2019).

84. M. Bota, O. Sporns, L. W. Swanson, Architecture of the cerebral cortical association connectome underlying cognition. Proc. Natl. Acad. Sci. U. S. A. 112, E2093-E2101 (2015).

85. E. M. Gordon, et al., Default-mode network streams for coupling to language and control systems. Proc. Natl. Acad. Sci. U. S. A. 117, 17308-17319 (2020).

86. C. McCormick, E. Ciaramelli, F. De Luca, E. A. Maguire, Comparing and contrasting the cognitive effects of hippocampal and ventromedial prefrontal cortex damage: A review of 
human lesion studies. Neuroscience (2017)

https:/doi.org/10.1016/j.neuroscience.2017.07.066.

1103 87. H. Kondo, P. Lavenex, D. G. Amaral, Intrinsic connections of the macaque monkey hippocampal formation: II. CA3 connections. J. Comp. Neurol. 377, NA-NA (2009).

1105 88. I. K. Brunec, et al., Multiple Scales of Representation along the Hippocampal Anteroposterior Axis in Humans. Curr. Biol. 28, 2129-2135.e6 (2018).

89. J. W. Vogel, et al., A molecular gradient along the longitudinal axis of the human hippocampus informs large-scale behavioral systems. Nat. Commun. 11, 1-17 (2020). Functional Connectivity in Temporal Lobe Epilepsy. Front. Neurol. 10, 1-12 (2019). axis. Hippocampus, 1-16 (2019).

1114 92. M. S. Fanselow, H. W. Dong, Are the Dorsal and Ventral Hippocampus Functionally 1115 Distinct Structures? Neuron 65, 7-19 (2010).

1116 93. I. R. Olson, D. McCoy, E. Klobusicky, L. A. Ross, Social cognition and the anterior 1117 temporal lobes: A review and theoretical framework. Soc. Cogn. Affect. Neurosci. 8, 123$1118133(2013)$.

1119 94. M. Cohn, M. St-Laurent, A. Barnett, M. P. McAndrews, Social inference deficits in 1120 temporal lobe epilepsy and lobectomy: Risk factors and neural substrates. Soc. Cogn. $1121 \quad$ Affect. Neurosci. 10, 636-644 (2015).

1122 95. L. M. DiNicola, R. M. Braga, R. L. Buckner, Parallel distributed networks dissociate episodic and social functions within the individual. J. Neurophysiol. 123, 1144-1179 
(2020).

1125 96. J. R. Andrews-Hanna, R. Saxe, T. Yarkoni, Contributions of episodic retrieval and

1126 mentalizing to autobiographical thought: Evidence from functional neuroimaging, resting-

1127 state connectivity, and fMRI meta-analyses. Neuroimage 91, 324-335 (2014).

1128 97. S. Lee, T. Parthasarathi, J. W. Kable, The dorsal and ventral default mode networks are

1129 dissociably modulated by the valence and vividness of imagined events. bioRxiv,

$1130 \quad 2020.05 .21 .109728(2020)$.

$113198 . \quad$ N. E. Nawa, H. Ando, Effective connectivity within the ventromedial prefrontal cortex1132 hippocampus-amygdala network during the elaboration of emotional autobiographical 1133 memories. Neuroimage 189, 316-328 (2019).

1134 99. N. Franzmeier, et al., Functional brain architecture is associated with the rate of tau 1135 accumulation in Alzheimer's disease. Nat. Commun. 11, 1-17 (2020).

1136 100. S. M. Hayes, D. H. Salat, M. Verfaellie, Default network connectivity in medial temporal 1137 lobe amnesia. J. Neurosci. 32, 14622-14630 (2012).

1138 101. J. D. Power, et al., Functional Network Organization of the Human Brain. Neuron 72, $1139 \quad 665-678(2011)$.

1140 102. E. M. Gordon, et al., Individual-specific features of brain systems identified with resting $1141 \quad$ state functional correlations. Neuroimage 146, 918-939 (2017).

1142 103. O. Esteban, et al., fMRIPrep: a robust preprocessing pipeline for functional MRI. Nat. $1143 \quad$ Methods 16, 111-116 (2019).

1144 104. O. Esteban, et al., fMRIPrep: a robust preprocessing pipeline for functional MRI (2019) 1145 https:/doi.org/10.5281/ZENODO.2859286 (March 31, 2020).

1146 105. K. Gorgolewski, et al., Nipype: A Flexible, Lightweight and Extensible Neuroimaging 
1147 Data Processing Framework in Python. Front. Neuroinform. 5 (2011).

1148 106. K. J. Gorgolewski, et al., nipy/nipype: 1.2 .0 (2019)

1149 https:/doi.org/10.5281/ZENODO.2685428 (March 31, 2020).

1150 107. R. W. Cox, J. S. Hyde, Software tools for analysis and visualization of fMRI data. NMR

$1151 \quad$ Biomed. 10, 171-8.

1152 108. M. Jenkinson, S. Smith, A global optimisation method for robust affine registration of 1153 brain images. Med. Image Anal. 5, 143-156 (2001).

1154 109. D. N. Greve, B. Fischl, Accurate and robust brain image alignment using boundary-based 1155 registration. Neuroimage 48, 63-72 (2009).

1156 110. M. Jenkinson, P. Bannister, M. Brady, S. Smith, Improved optimization for the robust and 1157 accurate linear registration and motion correction of brain images. Neuroimage 17, 825$1158 \quad 841(2002)$.

1159 111. C. Lanczos, Evaluation of noisy data. J. Soc. Ind. Appl. Math. Ser. B Numer. Anal. 1, 76$1160 \quad 85(1964)$.

1161 112. A. Abraham, et al., Machine learning for neuroimaging with scikit-learn. Front.

$1162 \quad$ Neuroinform. 8 (2014).

1163 113. R. Ciric, et al., Benchmarking of participant-level confound regression strategies for the 1164 control of motion artifact in studies of functional connectivity. Neuroimage 154, 174-187 1165 (2017).

1166 114. A. Weissenbacher, et al., Correlations and anticorrelations in resting-state functional 1167 connectivity MRI: A quantitative comparison of preprocessing strategies. Neuroimage 47, $1168 \quad 1408-1416(2009)$.

1169 115. J. D. Power, et al., Methods to detect, characterize, and remove motion artifact in resting 
state fMRI. Neuroimage 84, 320-341 (2014).

1171 116. M. Rubinov, O. Sporns, Weight-conserving characterization of complex functional brain

1172 networks. Neuroimage 56, 2068-2079 (2011).

1173 117. C. Gratton, et al., Functional Brain Networks Are Dominated by Stable Group and

1174 Individual Factors, Not Cognitive or Daily Variation. Neuron 98, 439-452.e5 (2018).

1175 118. B. A. Seitzman, et al., A set of functionally-defined brain regions with improved

1176 representation of the subcortex and cerebellum. Neuroimage 206, 116290 (2020).

1177 119. M. A. Bertolero, B. T. T. Yeo, D. S. Bassett, M. D’Esposito, A mechanistic model of

1178 connector hubs, modularity and cognition. Nat. Hum. Behav. 2, 765-777 (2018).

1179 120. S. Seabold, J. Perktold, Statsmodels: econometric and statistical modeling with python.

1180 Proc. 9th Python Sci. Conf. , June 28 - July 3, Austin, Texas, 57-61 (2010).

1181 121. H. Nili, et al., A Toolbox for Representational Similarity Analysis. PLoS Comput. Biol.

$1182 \quad \mathbf{1 0}$, e1003553 (2014). 